\title{
Benzodithiophenedione-based polymers: recent advances in organic photovoltaics
}

\author{
Bing Zheng', Lijun Huo $\mathbb{D}^{1}$ and Yongfang Li ${ }^{2}$
}

\begin{abstract}
Over the past 20 years, significant progress has been made in organic photovoltaics (OPVs) due to its advantages of being cost-effective, being lightweight, and having flexible manufacturability. The optical-active layer of OPVs consists of a $p$-type polymer as the donor and an $n$-type small molecule as the acceptor. An efficient design strategy of a polymer donor is based on an alternating electron-donating unit (D) and an electron-accepting unit (A). Among numerous electron-accepting units, an emerging annelated thiophene of benzodithiophenedione (BDD) has exhibited a distinguished photovoltaic performance because of its planar molecular structure, low-lying highest occupied molecular orbit (HOMO) level and good self-assembly property. In this review article, we summarize the most recent developments in BDD-based photovoltaic materials. Special attention is paid to the chemical structure-property relationships, such as the absorption, bandgap, energy levels, mobilities, and photovoltaic performances. The empirical regularities and perspectives on the future development of BDD-based photovoltaic materials are included.
\end{abstract}

\section{Introduction}

Organic photovoltaic (OPV) technology exhibits highly attractive features in realizing large areas, being lightweight, being low-cost and possessing flexible photovoltaic panels compared to traditional silicon-based photovoltaic cells. Currently, bulk heterojunction (BHJ)type OPVs are the most efficient device structures, in which the optical-active layer is composed of an interpenetrating network of electron donor materials and electron acceptor materials ${ }^{1,2}$. An encouraging state-ofthe-art device has exhibited power conversion efficiencies (PCEs) over 17\%, which represents a crucial step toward the commercialization of $\mathrm{OPVs}^{3}$. Among the rapid development of optical-active donor/acceptor materials, interface materials and device engineering, the properties of donors and acceptors, such as absorption, energy levels, and bandgaps, play a vital role in realizing high PCEs. Therefore, the evolution of new photovoltaic materials with a smaller optical bandgap $\left(E_{\mathrm{g}}\right)$ and reasonable highest

Correspondence: Lijun Huo (huolijun@buaa.edu.cn)

'School of Chemistry, Beihang University, 100191 Beijing, PR China

${ }^{2}$ CAS Key Laboratory of Organic Solids, Institute of Chemistry, Chinese

Academy of Sciences, 100190 Beijing, PR China occupied molecular orbital (HOMO) and lowest unoccupied molecular orbital (LUMO) levels is crucial to further improve the PCEs of OPVs.

During the earlier development period of organic semiconductors for OPVs, a classic conjugated unit of thiophene dominated the construction of efficient donor materials. Polythiophene (PTh) and its derivatives in particular have been extensively studied due to their good $\pi$-electron delocalization property and high thermal stability $^{4}$ (Fig. 1). For example, blends of poly(3-hexylthiophene) (P3HT) and [6,6]-phenyl- $\mathrm{C}_{61}$-butyric acid methyl ester $\left(\mathbf{P C}_{\mathbf{6 1}} \mathbf{B M}\right)$ remain among the most interesting candidates in the OPV field. A large number of papers have explored the effects of the degree of regioregularity and order of PTh on the photovoltaic performance. The PCEs based on PTh have increased step by step from $0.6 \%$ up to $7.4 \%$ across $2001-2012^{5}$. Despite the rapid progress in the development of additional new acceptors reported after that time, the photovoltaic performance based on P3HT has not improved due to the original inferiority of P3HT in its mismatched absorption ranges and relatively higher-lying HOMO energy level. To overcome these disadvantages of $\mathrm{PTh}$, donor-acceptor 


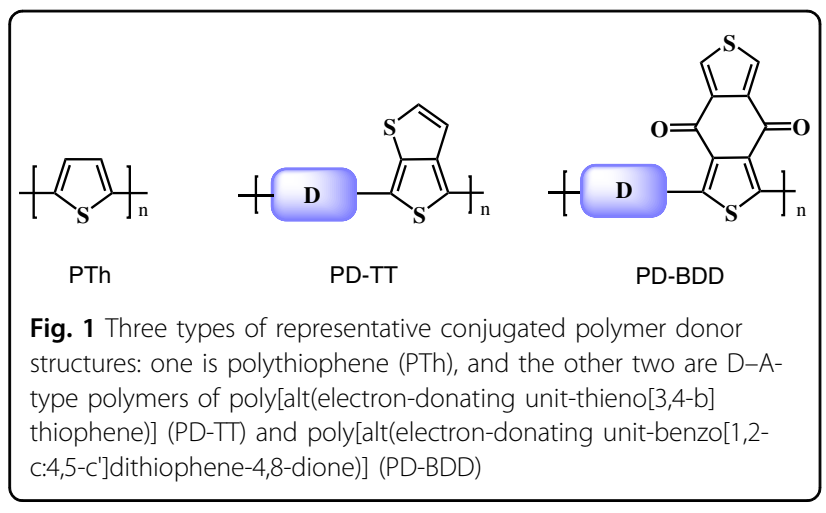

(D-A)-type conjugated polymers as donors were thus extensively developed for OPVs because the hybridization of the electron-donating and electron-accepting molecular orbitals in D-A polymers typically leads to a tunable bandgap and energy level. Meanwhile, the adoption of a fused-ring thiophene derivative of thieno[3,4-b]thiophene (TT) with an electron-withdrawing side chain as an electron-accepting unit to construct a D-A polymer has become popular for increasing PCEs ${ }^{6}$. In 2008, Yu et al. first reported a low-bandgap photovoltaic polymer based on TT with an electron-withdrawing side chain as the acceptor unit ${ }^{7}$. The unique wide-range absorption property of TT has inspired many novel low-bandgap polymer donors. In comparison with PTh as a donor, the TT-based D-A polymer of poly[alt(electron-donating unit-thieno [3,4-b]thiophene) (PD-TT) has accelerated the improvement of PCEs in the past 10 years. The PCEs based on PD-TT were promoted from $1.9 \%$ in 2008 to $13.8 \%$ at the end of $2018^{8}$. Therefore, the adoption of a fused-ring thiophene derivative as an electron-accepting unit provides a successful strategy for the development of OPV donors. Some typical fused-ring thiophene derivatives as electron-accepting units have been synthesized and applied in OPVs, such as thieno[3,4-c]pyrole-4,6dione $(\mathrm{TPD})^{9}$, difluorodioxocyclopenta-[c]thiophene $(\mathrm{DCT})^{10}$, benzo[c]thiophene (named isothianaphthene, ITN $)^{11,12}$, and so on, which become useful A units in constructing efficient D-A-type photovoltaic polymer donors.

In recent years, another non-classical annelated thiophene of benzo[1,2-c:4,5-c']dithiophene-4,8-dione (BDD) has emerged in polymer donors as an electron-accepting core due to its planar molecular structure, low-lying HOMO level and good self-assembly property. Compared to the development of PTh and PD-TT polymers, rapid and incredible progress has been made in the BDD-based polymer donor in the past 7 years (Fig. 2). The first PCE of $4.5 \%$ reported for the PSCs with the BDD-based polymer as a donor in 2012 ascended rapidly up to over 15\% in 2019, which gives an impressive new PCE record in

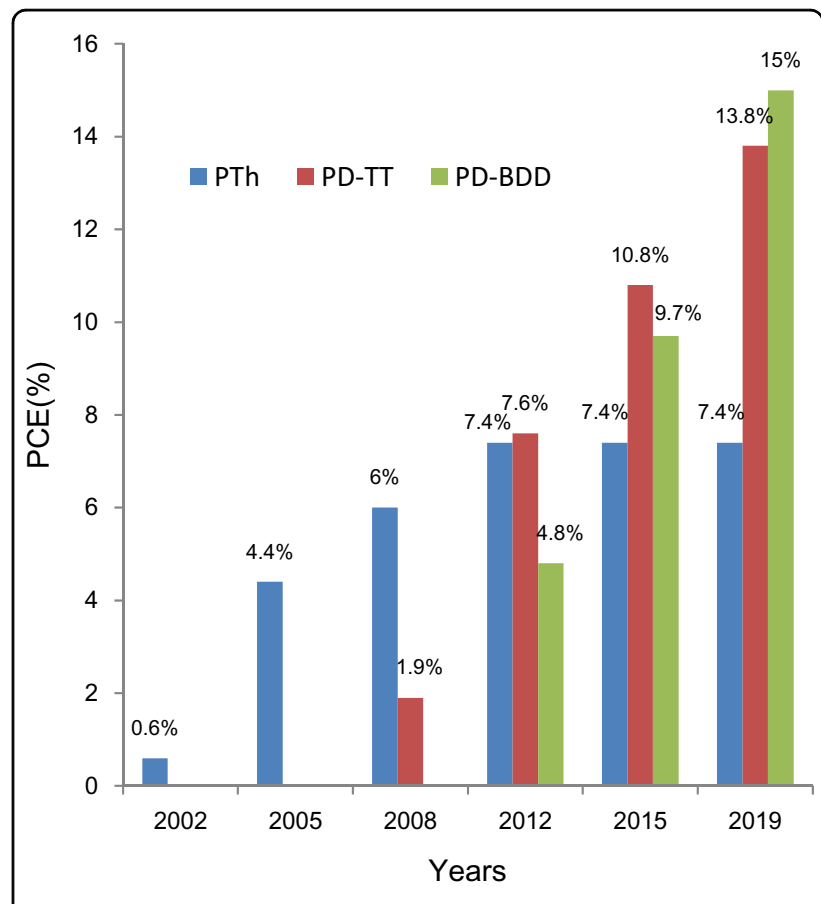

Fig. 2 Research progress with regard to the efficiency enhancement for PTh, PD-TT and PD-BDD systems from 2001-2019

single-junction OPV devices ${ }^{13}$. Although there have been so many reviews on the donor of OPVs in the past few years, many of them have focused on the well-known PTh and PD-TT systems. To cover the latest developments, we will review recent studies on the new PD-BDD system. These new donor materials in the PD-BDD system have attracted much interest because of their promising photovoltaic performance for commercial applications. Based on the material numbers applied in one optical-active layer, binary, ternary, and tandem components containing BDD as the electron-accepting unit are discussed, and we focus on the chemical structure-property relationships, such as the absorption, bandgap, energy levels, mobilities, and photovoltaic performances. The empirical regularities and perspectives on the future development of BDD-based photovoltaic materials are also included.

The starting material of (1) was prepared by adding excessive oxalyl chloride in methylene chloride solution under ambient temperature overnight. The intermediate of (2) was finished by dropping lithium diisopropylamide (LDA) into thiophene solution at ambient temperature, and then alkylbromide was added and stirred overnight. Then, 1,3-dibromo-5,7-dihexyl-4H,8H-benzo [1,2-c:4,5-c] dithiophene-4,8-dione (3) was prepared by Friedel-Crafts acylation of 2,5-alkylthiophene (2) with 2,5-dibromo-3,4thiophenedicarbonyl dichloride (1) according to the procedure reported ${ }^{14}$. The Stille coupling reactions between 


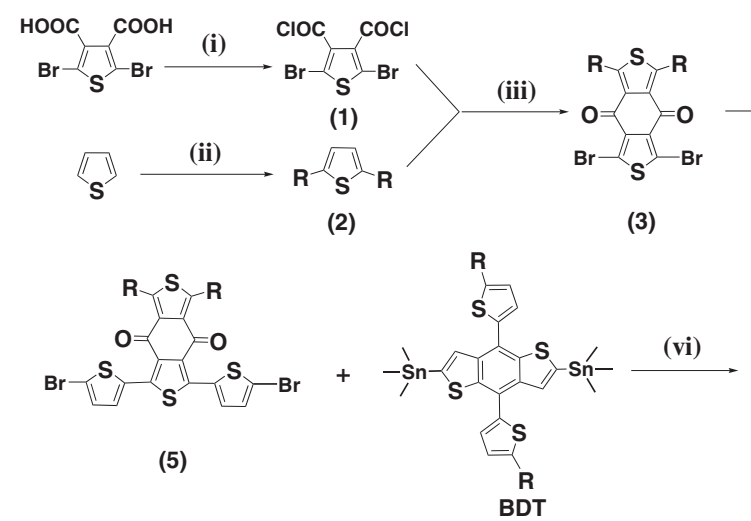

(iv)

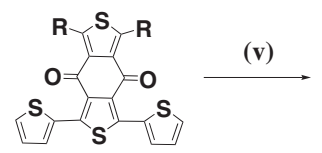

(4)

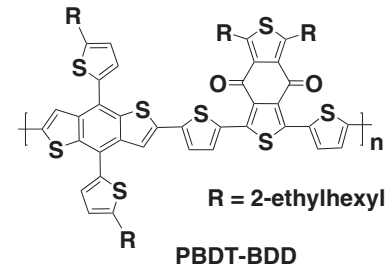

Scheme 1 Synthetic procedures of a typical BDD unit and its corresponding copolymer. (i) Oxalyl chloride, methylene chloride, ambient temperature, overnight; (ii) lithium diisopropylamide, THF, alkylbromide, ambient temperature, overnight; (iii) $\mathrm{AlCl}_{3}$, methylene chloride, ambient temperature, then reflux for several hours; (iv) 2-(tributylstannyl)thiophene, toluene, $\mathrm{Pd}\left(\mathrm{PPh}_{3}\right)_{4}$, reflux, overnight; (v) $\mathrm{Br}_{2}$, chloroform, ambient temperature, overnight; (vi) toluene, $\mathrm{Pd}\left(\mathrm{PPh}_{3}\right)_{4}$, reflux, $12 \mathrm{~h}$

brominated compounds (3) and 2-(tributylstannyl)thiophene in the presence of $\mathrm{Pd}\left(\mathrm{PPh}_{3}\right)_{4}$ in toluene at $120^{\circ} \mathrm{C}$ overnight to give the intermediate of (4). Subsequently, a distannylated compound (such as BDT) was reacted with the bromide of (5) with a catalyst of $\mathrm{Pd}\left(\mathrm{PPh}_{3}\right)_{4}$ to obtain the target copolymer (such as PBDT-BDD) (Scheme 1) ${ }^{15}$. BDD-based polymers exhibit several advances, such as high thermal stability, low-lying HOMO levels, and high mobility. For example, for a BDD-based copolymer of PBDT-BDD, it was reported that the polymer exhibited good thermal stability below $350^{\circ} \mathrm{C}$ under an inert atmosphere. The most interesting character of PBDT-BDD is that it showed a planar molecular conformation due to small twist angles between the adjacent thiophene $\pi$-bride and BDD unit and between the BDT and $\pi$-bride (Fig. 3a). Consequently, a typical temperature-dependent-aggregation (TDA) behavior was observed in its solution and rendered a pronounced aggregation in its thin film (Fig. 3c, d), which could realize enhanced crystallinity and $\pi-\pi$ stacking and high mobility. In addition, a low HOMO level of $-5.23 \mathrm{eV}$ and a wide bandgap of $2.05 \mathrm{eV}$ were realized due to the strong electron-drawing property of carbonyl groups attached to the BDD unit (Fig. 3b). The combination of all these optical-electronic properties exhibits potential advantages in constructing highperformance polymer donors.

\section{BDD-based polymer donors used in fullerene- acceptor-based OPVs}

The typical conventional device architecture of an OPV is shown in Fig. 4, where the active layer (donor and acceptor blend) is defined by an interpenetrating network of donor and acceptor components sandwiched between different work function electrodes (such as $\mathrm{Al}$ and ITO). The overall device architecture is classified as either fullerene- or nonfullerene-based OPVs. Here, we first focus on the composition of the donor and fullerene-based (such as PCBM) binary system. The polymeric structures are shown in Fig. 5, and the optical-electronic properties and the related photovoltaic performances of all the copolymers are summarized in Table 1.

The BDD unit as an electron-accepting core was first reported in 2012 for OPVs by Y. Ie and Y. Aso, and a D-A copolymer P1 was synthesized by copolymerization of BDD and dithieno[3,2-b:2', 3'-d] silole (DTS). The OPV with $\mathbf{P 1}$ as the donor and PCBM as the acceptor preliminarily exhibited higher open-circuit voltage $\left(V_{\text {oc }}\right)$ values $(0.90-0.98 \mathrm{~V})$ than those of typical P3HT-based devices $(\mathrm{ca} .0 .6 \mathrm{~V})^{16}$. This result can be attributed to the natural character of the BDD-based polymers with lowlying HOMO levels. Meanwhile, the alternating D-A copolymer P2 of benzodithiophene (BDT) and BDD showed a poor PCE of less than 1\% compared to P1, with a PCE of $2.7 \%$. It was found that P1 formed a smooth morphology, while P2 formed large domain sizes and obvious phase separation. In addition, a higher shortcircuit current $\left(J_{\text {sc }}\right)$ value of P1 than that of P2 is attributed to the higher hole mobility of the former than that of the latter. Since then, numerous scientists have focused on designing and synthesizing novel polymers based on BDD units. Some BDD-based polymers exhibit fascinating optical, electronic and photovoltaic properties. The optoelectronic properties of these polymers as donors in the OPVs with the fullerene derivative as the acceptor discussed throughout this article are summarized in Table 1.

The BDT unit with thiophene conjugated side chains (BDTT), first reported by Huo et al., is a key donor unit 
(a)

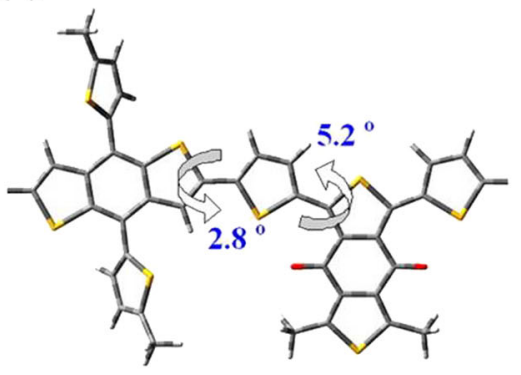

(c)

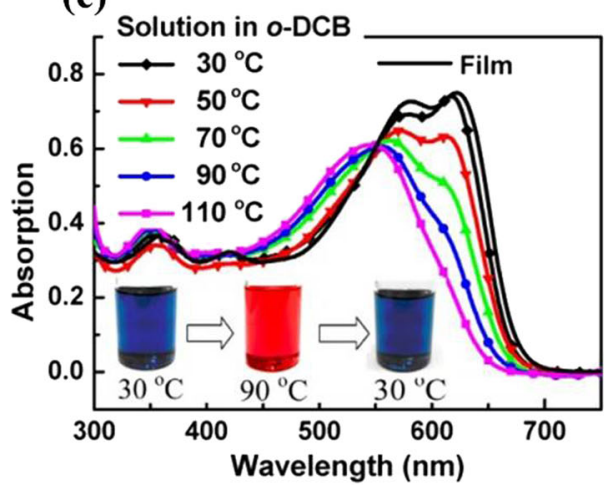

(b)
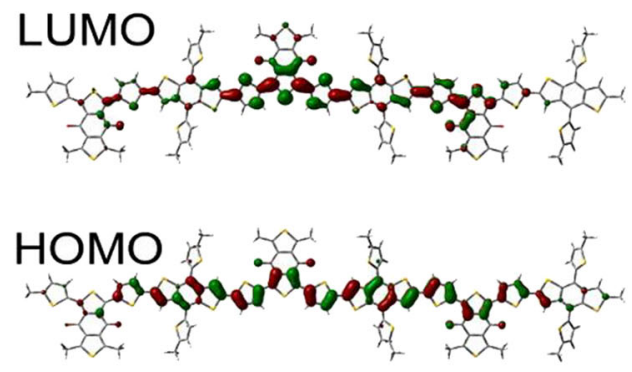

(d)

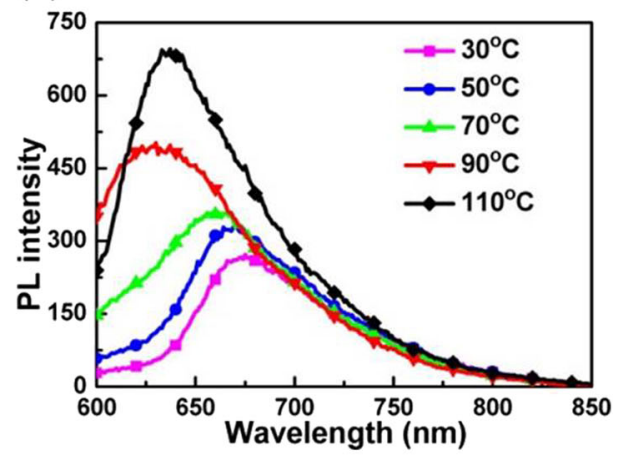

Fig. 3 a Molecular conformation and $\mathbf{b}$ the frontier molecular orbital surfaces of PBDT-BDD; $\mathbf{c}$ UV-vis spectra of PBDT-BDD film and in o-DCB solution under different temperatures (inset: the color of the solution under different temperatures); $\mathbf{d}$ temperature-dependent photoluminescence spectra of P3 in o-DCB. Reproduced with permission ${ }^{15}$. Copyright 2012 American Chemical Society

in constructing high-performance polymer donors. Hou et al. synthesized a D-A copolymer P3 by copolymerization of the BDTT donor unit and BDD acceptor unit $^{15}$. After optimizing the device heating conditions, a high charge mobility and a PCE of $6.67 \%$ were recorded for the P3-based device, which provides an example to reveal the correlation between the strong aggregation character and morphology of the blend films. To further extend the conjugated degree in the BDD-based polymeric main backbones, Huo et al. introduced a larger $\pi$-conjugated bridge of bithophene and thienothiophene to replace thiophene $\pi$-bridges and synthesized P4 and P5, respectively ${ }^{17}$. Compared with P5, P4 showed a deeper HOMO and a more planar backbone structure with slightly higher mobility. Correspondingly, P4 gave a higher PCE (6.93\%) than that of P5 (3.92\%). The results indicate that adopting a reasonable and effective large $\pi$-bridge of the polymer backbone enhances the photovoltaic performance. In contrast with the extended conjugated degree by $\pi$ bridge, Huo et al. introduced a more efficient method by designing a high linear building block of dithieno[2,3$\mathrm{d} ; 2^{\prime}, 3^{\prime}$ - $\mathrm{d}^{\prime}$ ] benzo[1,2-b;4,5-b']dithiophene (DTBDT) and synthesized polymer $\mathbf{P 6}{ }^{18}$. $\mathbf{P 6}$ with an extended $\pi$-conjugation in the BDT unit could effectively improve its charge carrier mobilities and rendered an impressive PCE of $9.74 \%$, which is one of the highest PCE values among the OPVs based on a wide-bandgap polymeric donor with a fullerene acceptor. This is mainly due to its more planar linear molecular conformation, which reduces the conformational disorder of the backbone and increases the molecular rigidity of the polymer in the solid state and can facilitate electron delocalization.

Except for the two-dimensional conjugated strategy, another efficient strategy of varying the heteroatom on conjugated building blocks has also shown its fascinating advances in adjusting the optical-electronic properties of polymers. For example, compared to their Si-bridged DTS analogues, the dithieno[3,2-b:2', $3^{\prime}$-d] germole (DTG) unit with the more ordered crystalline structures was successfully applied in BDD-based polymers. The P7 copolymer consisting of DTG and BDD units reported by Hou et al. showed a higher $J_{\mathrm{sc}}$ and PCE than its analogue $\mathbf{P 1}^{19}$. The improvement of the efficiency was attributed to the higher hole mobility of the DTG-based polymers and the more favorable phase-separation morphology of the blend films. Huo et al. conducted a series of studies on BDD-based heteroatom polymers. They adopted benzodifuran (BDF) to replace BDT as an electron-rich unit 


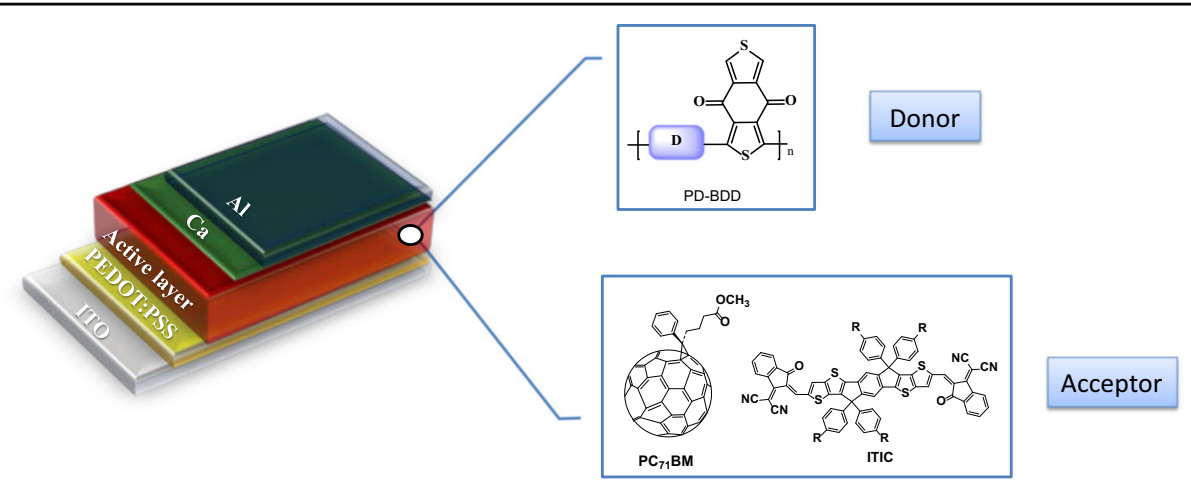

Fig. 4 Conventional device architecture of OPVs with BDD-based polymer as the electron donor and fullerene derivative PCBM or nonfullerene n-type organic small molecule ITIC as the electron acceptor

because the oxygen atom, with a smaller atom size than that of the sulfur atom, can improve the planarity of repeating units in polymeric backbones and strengthen intermolecular interactions. As a result, phenylsubstituted BDF polymer P8 showed a high PCE of $9.43 \%$, with $J_{\mathrm{sc}}$ of $13.28 \mathrm{~mA} \mathrm{~cm}^{-2}, V_{\mathrm{oc}}$ of 0.92 , and FF of $77.4 \%$, which were higher than those of its BDT counterpart $\mathbf{P 9 ^ { 2 0 }}$. A deep HOMO level of $-5.43 \mathrm{eV}$ and a high hole mobility of $0.014 \mathrm{~cm}^{2} \mathrm{~V}^{-1} \mathrm{~s}^{-1}$ can explain the high PCEs of P8 over P9. Huo et al. then investigated the effect of 2D-BDT side chain heteroatoms (thiophene, furan and selenophene) on the photovoltaic performance of polymer donors. Both P11 and P12 exhibited a higher PCE of $7.48 \%$ and $8.52 \%$, respectively, than that of P10, due to the high crystallinity and preferential face-on orientation of the former compared with the latter ${ }^{21}$. Meanwhile, Huo et al. designed a group of polymers by inserting heteroatoms $(\mathrm{O}, \mathrm{S}$, and $\mathrm{Se})$ into the electron-accepting BDD unit to obtain P13-P15 ${ }^{22}$. Among these three polymers, the moderate crystallinity and aggregation of P14 exhibited the best photovoltaic performance, which is coincident with its balanced electron/hole ratio and best morphology among these blends. These results indicate the important role of the same main-group element engineering in tuning optical-electronic properties, including the absorption, energy level, crystallinity, mobility, and photovoltaic properties.

Apart from polymeric main backbone modifications, the alkyl side chain geometry should be taken into account because it not only could adjust the solubility of the polymer but also the position, length, and bulkiness of alkyl side chains will largely influence the aggregation behavior, including intermolecular interactions and related stacking/packing in the solid state. Much effort has been devoted to modifying BDD-based polymer side chains. Huo et al. designed a series of novel copolymers P16-P18 consisting of identical backbones with identical side chain carbon atom numbers but different geometries in $\mathrm{BDT}$ or $\mathrm{BDD}^{23}$. They exhibited obvious differences in the electronic structures, molecular packing, charge transport, and photovoltaic properties. The alkyl chain length on the BDD unit is very sensitive to the molecular geometry and induces different steric hindrances. Consequently, P17, with a moderate bulky side chain on the BDD unit, showed the best photovoltaic performance, with high PCEs over $10 \%$. Considering the overaggregated negative effects on the large phaseseparation degree and limited microphase interfaces in the blend, Huo et al. and Liang et al. introduced functional methoxyl groups to polymer side chains of P19 and P20, respectively. Both polymers showed a deeper HOMO level and better PCE than their counterparts, which was attributed to good crystallinity and higher domain purity in their blend films ${ }^{24,25}$. These results provide a facile way to penetrate overaggregations on polymer and blend films and to realize reasonable morphology. Hou et al. reported dialkylthio-substituted thienyl-benzodithiophene P21 to modulate the molecular levels of the polymers ${ }^{26}$. The $V_{\mathrm{oc}}$ of the P21-based device is as high as $1.0 \mathrm{~V}$, which is ca. $0.15 \mathrm{~V}$ higher than those of the corresponding alky-substituted analogues. Meanwhile, Zhang et al. reported two D-A copolymers P22 and P23 based on meta-positioned alkoxyphenyl side-chain-substituted BDT, in which selenylophene and thiophene unit as the $\pi$-bridge were incorporated into the main backbones ${ }^{27}$. Finally, both P22 and P23 showed high $V_{\text {oc }}$ over $0.9 \mathrm{~V}$ due to the deep HOMO level caused by some steric hindrances in metapositioned alkoxyphenyl side chains.

Generally, for most polymer donors, use of the donor$\pi$-acceptor $(D-\pi-A)$ architecture is an efficient strategy for adjusting their molecular energy levels, absorption spectra and charge mobilities. Thus, the exploration of new electron-donating units, electron-accepting units, and $\pi$-bridges toward efficient donor materials is popular 


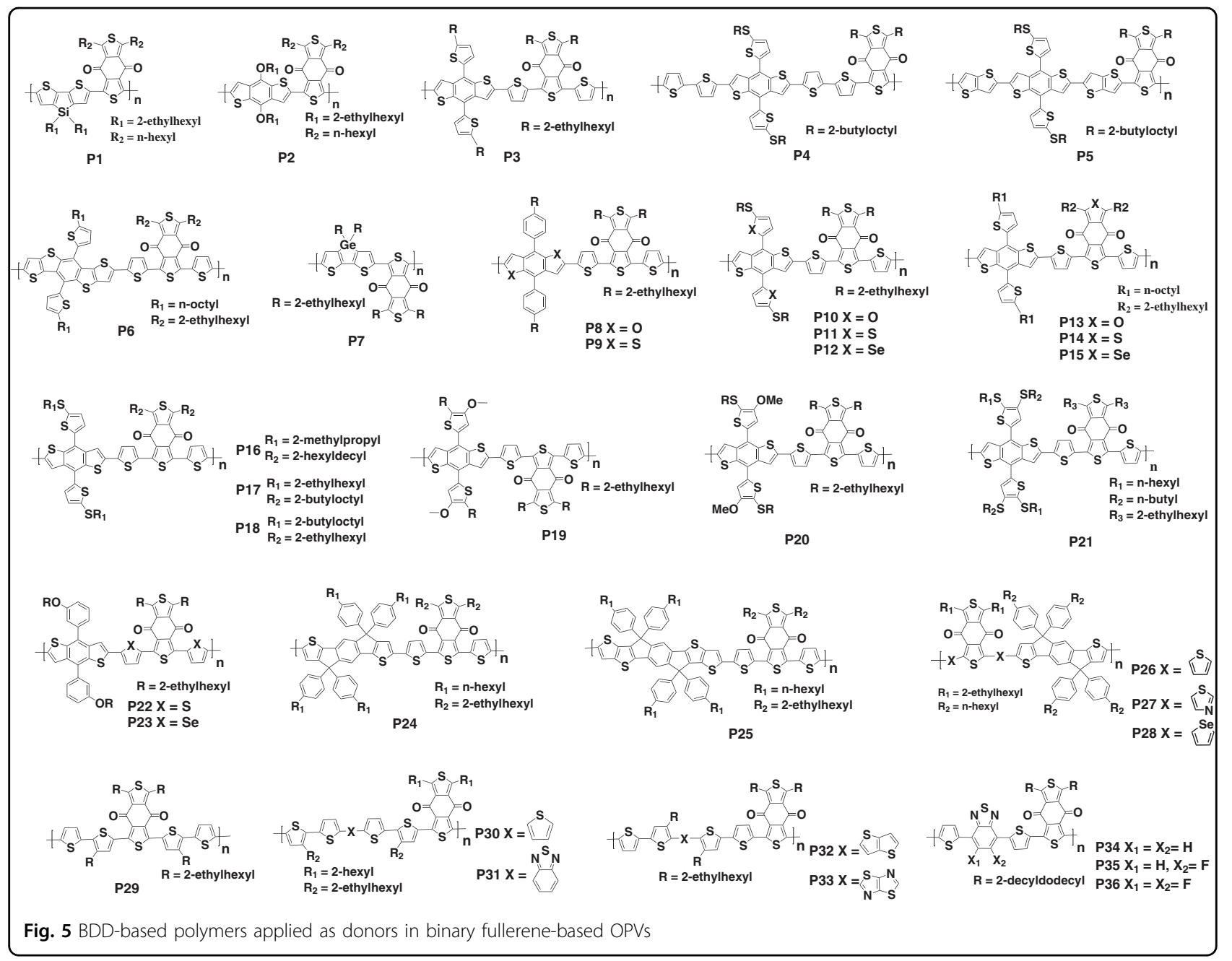

in organic semiconductors. Indacenodithiophene (IDT) and its derivatives (such as indacenodithieno[3,2-b]thiophene (IDTT)) represent another kind of conjugated fused-ring molecule with low-lying HOMO levels and high mobility. Huo et al. first copolymerized IDT/IDTT with BDD units to obtain P24 and P25, respectively ${ }^{28}$. The resulting P24 and P25 copolymers exhibited a comparatively low-lying HOMO level, and the latter, with an extended fused ring, was found to have better coplanarity and higher carrier mobility $\left(0.11 \mathrm{~cm}^{2} \mathrm{~V}^{-1} \mathrm{~s}^{-1}\right)$, which resulted in a higher PCE. Furthermore, Sun et al. varied $\pi$-bridges of thiophene, selenophene and thiazole units to comprehensively evaluate the $\pi$-bridge effects. Compared to P26, both P27 and P28 exhibited enhanced intermolecular interactions, an expanded absorption range and better molecular planarity ${ }^{29}$. Among these three copolymers, P27 showed the highest PCE (8.65\%). However, P28 demonstrated a rather low efficiency of $2.75 \%$, which is due to the inferior photocurrent and fill factor caused by the poor exciton dissociation and low hole and electron mobilities due to some defects in the thiazole $\pi$-bridge. In addition, oligothiophene and a heteroatom fused ring were also used to replace BDT or IDT units as electron-donating units. Hou et al. reported that P29 with bithiophene as the D unit achieved a modest $\mathrm{PCE}^{30}$. It was interesting that from the grazingincidence X-ray diffraction (GIXD), P29 showed an unusual small lamellar space of $15.7 \AA$, which was a very small lamellar spacing compared to that of P3HT $\left(\begin{array}{l}16.7 \AA \\ \AA\end{array}\right)$. To further extend the absorption range to harvest more sunlight, Huo and Sun et al. synthesized two polymers: P30 and P31, inserting more thiophene or benzodithiadiazole (BT) on the main backbones to enlarge the absorption range ${ }^{31}$. In comparison with P30, P31 showed broader absorption spectra, a higher absorption coefficient, and correspondingly a higher PCE. They also compared the thieno[3,2-b]thiophene and thiazolo[5,4-d]thiazole polymers P32 and P33 polymers $^{32}$. The higher performance of P33 was mainly ascribed to the higher crystallinity and domain purity of 
Table 1 Polymer characteristics and the corresponding device performances of fullerene-based binary OPVs

\begin{tabular}{|c|c|c|c|c|c|c|c|c|c|}
\hline Donor & $E_{\mathrm{g}}^{\mathrm{opt}}(\mathrm{eV})$ & HOMO/ LUMO (eV) & Acceptor & $\mu_{\mathrm{h}}\left(\mathrm{cm}^{2} \cdot \mathrm{v}^{-1} \cdot \mathrm{s}^{-1}\right)$ & $J_{s c}\left(m A c m^{-2}\right)$ & $V_{\text {oc }}(\mathrm{V})$ & FF (\%) & PCE (\%) & ref \\
\hline P1 & 1.72 & $-5.33 /-3.39$ & $P C_{61} B M$ & $3.2 \times 10^{-5}$ & 6.01 & 0.98 & 46.0 & 2.70 & 16 \\
\hline P2 & 1.92 & $-5.41 /-3.45$ & $\mathrm{PC}_{61} \mathrm{BM}$ & $5.7 \times 10^{-7}$ & 2.64 & 0.87 & 33.0 & 0.73 & 16 \\
\hline P3 & & $-5.23 /-3.18$ & $P C_{61} B M$ & & 10.68 & 0.869955 & 72.277 & 6.67 & 15 \\
\hline P4 & 1.81 & $-5.86 /-3.89$ & $P C_{71} B M$ & $1.62 \times 10^{-3}$ & 11.0 & 0.86 & 72.9 & 6.93 & 17 \\
\hline P5 & 1.82 & $-5.74 /-3.87$ & $P C_{71} B M$ & $1.17 \times 10^{-3}$ & 8.60 & 0.85 & 53.1 & 3.92 & 17 \\
\hline P6 & 1.85 & $-5.36 /-3.43$ & $P C_{71} B M$ & & 14.11 & 0.92 & 75.0 & 9.74 & 18 \\
\hline P7 & 1.65 & $-5.41 /-3.76$ & $P C_{71} B M$ & $1.17 \times 10^{-4}$ & 10.30 & 0.9355 & 65.0 & 6.28 & 19 \\
\hline P8 & 1.83 & $-5.43 /-3.54$ & $P C_{71} B M$ & $1.4 \times 10^{-3}$ & 13.28 & 0.9255 & 77.4 & 9.43 & 20 \\
\hline P9 & 1.81 & $-5.32 /-3.52$ & $P C_{71} B M$ & $1.0 \times 10^{-4}$ & 13.13 & 0.8655 & 71.8 & 8.12 & 20 \\
\hline P10 & 1.74 & $-5.31 /-3.45$ & $P C_{71} B M$ & $3.13 \times 10^{-5}$ & 9.60 & 0.8355 & 49.5 & 4.01 & 21 \\
\hline P11 & 1.81 & $-5.52 /-3.56$ & $P C_{71} B M$ & $5.30 \times 10^{-3}$ & 12.20 & 0.8955 & 67.7 & 7.48 & 21 \\
\hline P12 & 1.80 & $-5.53 /-3.59$ & $P C_{71} B M$ & $1.34 \times 10^{-3}$ & 13.00 & 0.9155 & 71.7 & 8.52 & 21 \\
\hline P13 & 1.82 & $-5.51 /-3.52$ & $\mathrm{PC}_{71} \mathrm{BM}$ & $1.2 \times 10^{-4}$ & 9.30 & 0.8655 & 63.3 & 5.20 & 22 \\
\hline P14 & 1.81 & $-5.53 /-3.57$ & $P C_{71} B M$ & $2.9 \times 10^{-4}$ & 13.40 & 0.9055 & 74.2 & 9.00 & 22 \\
\hline P15 & 1.80 & $-5.46 /-3.53$ & $P C_{71} B M$ & $2.4 \times 10^{-4}$ & 12.90 & 0.8555 & 73.6 & 8.30 & 22 \\
\hline P16 & 1.86 & $-5.49 /-3.46$ & $P C_{71} B M$ & $1.24 \times 10^{-3}$ & 12.81 & 0.9755 & 72.2 & 9.10 & 23 \\
\hline P17 & 1.84 & $-5.43 /-3.43$ & $P C_{71} B M$ & $1.94 \times 10^{-3}$ & 14.14 & 0.9655 & 74.7 & 10.30 & 23 \\
\hline P18 & 1.81 & $-5.40 /-3.41$ & $P C_{71} B M$ & $1.25 \times 10^{-3}$ & 12.97 & 0.9255 & 67.2 & 8.10 & 23 \\
\hline P19 & 1.82 & $-5.16 /-3.11$ & $P C_{71} B M$ & $2.5 \times 10^{-5}$ & 12.45 & 0.8455 & 65.0 & 6.77 & 24 \\
\hline P20 & 1.81 & $-5.65 /-3.55$ & $P C_{71} B M$ & $1.4 \times 10^{-3}$ & 12.80 & 0.9855 & 71.4 & 9.20 & 25 \\
\hline P21 & 1.83 & $-5.52 /$ & $P C_{71} B M$ & $1.28 \times 10^{-3}$ & 10.80 & 1.0055 & 55.1 & 5.95 & 26 \\
\hline P22 & 1.90 & $-5.42 /-3.36$ & $\mathrm{PC}_{71} \mathrm{BM}$ & $5.76 \times 10^{-4}$ & 12.40 & 0.9555 & 71.0 & 8.40 & 27 \\
\hline P23 & 1.77 & $-5.35 /-3.31$ & $\mathrm{PC}_{71} \mathrm{BM}$ & $6.22 \times 10^{-4}$ & 14.90 & 0.9055 & 73.0 & 9.80 & 27 \\
\hline P24 & 1.90 & $-5.26 /-3.48$ & $P C_{71} B M$ & $2.49 \times 10^{-3}$ & 10.78 & 0.9255 & 62.5 & 6.20 & 28 \\
\hline P25 & 1.90 & $-5.29 /-3.50$ & $P C_{71} B M$ & $3.05 \times 10^{-3}$ & 11.25 & 0.9255 & 62.2 & 6.44 & 28 \\
\hline P26 & 1.85 & $-5.62 /-3.77$ & $P C_{71} B M$ & $4.28 \times 10^{-4}$ & 11.38 & 0.9155 & 68.0 & 7.04 & 29 \\
\hline P27 & 1.76 & $-5.40 /-3.64$ & $P C_{71} B M$ & $2.43 \times 10^{-4}$ & 16.04 & 0.899955 & 60.0 & 8.65 & 29 \\
\hline P28 & 1.86 & $-5.67 /-3.81$ & $P C_{71} B M$ & $0.31 \times 10^{-4}$ & 8.12 & 0.96855 & 35.0 & 2.75 & 29 \\
\hline P29 & & $-5.13 /-3.23$ & $P C_{71} B M$ & & 11.57 & 0.83855 & 71.0 & 6.88 & 30 \\
\hline P30 & 1.72 & $-5.34 /-3.56$ & $P C_{71} B M$ & $4.77 \times 10^{-3}$ & 9.16 & 0.819955 & 61.4 & 4.70 & 31 \\
\hline P31 & 1.59 & $-5.38 /-3.62$ & $P C_{71} B M$ & $3.11 \times 10^{-4}$ & 12.15 & 0.72855 & 73.0 & 6.58 & 31 \\
\hline P32 & 1.79 & $-5.19 /-3.06$ & $\mathrm{PC}_{71} \mathrm{BM}$ & $0.77 \times 10^{-3}$ & 8.69 & 0.709955 & 55.0 & 3.40 & 32 \\
\hline P33 & 1.78 & $-5.31 /-3.19$ & $P C_{71} B M$ & $3.44 \times 10^{-3}$ & 12.87 & 0.66855 & 74.0 & 8.10 & 32 \\
\hline P34 & 1.68 & $-5.57 /-3.89$ & $P C_{71} B M$ & $3.67 \times 10^{-3}$ & 2.55 & 0.86855 & 60.3 & 1.40 & 33 \\
\hline P35 & 1.70 & $-5.62 /-3.92$ & $\mathrm{PC}_{71} \mathrm{BM}$ & $1.86 \times 10^{-3}$ & 2.96 & 0.939955 & 57.8 & 1.70 & 33 \\
\hline P36 & 1.73 & $-5.67 /-3.94$ & $P C_{71} B M$ & $4.92 \times 10^{-3}$ & 8.96 & 0.99855 & 67.6 & 6.20 & 33 \\
\hline
\end{tabular}

P33 over that of P32 in the blends. In addition, Moon et al. showed a series of copolymers P34-P36 ${ }^{33}$. By varying the number of fluorine $(F)$ atoms on the
BT unit, the bifluorination of P36 exhibited a decreased HOMO level, a predominant face-on orientation and subsequently a higher PCE of $6.2 \%$ than those based on 


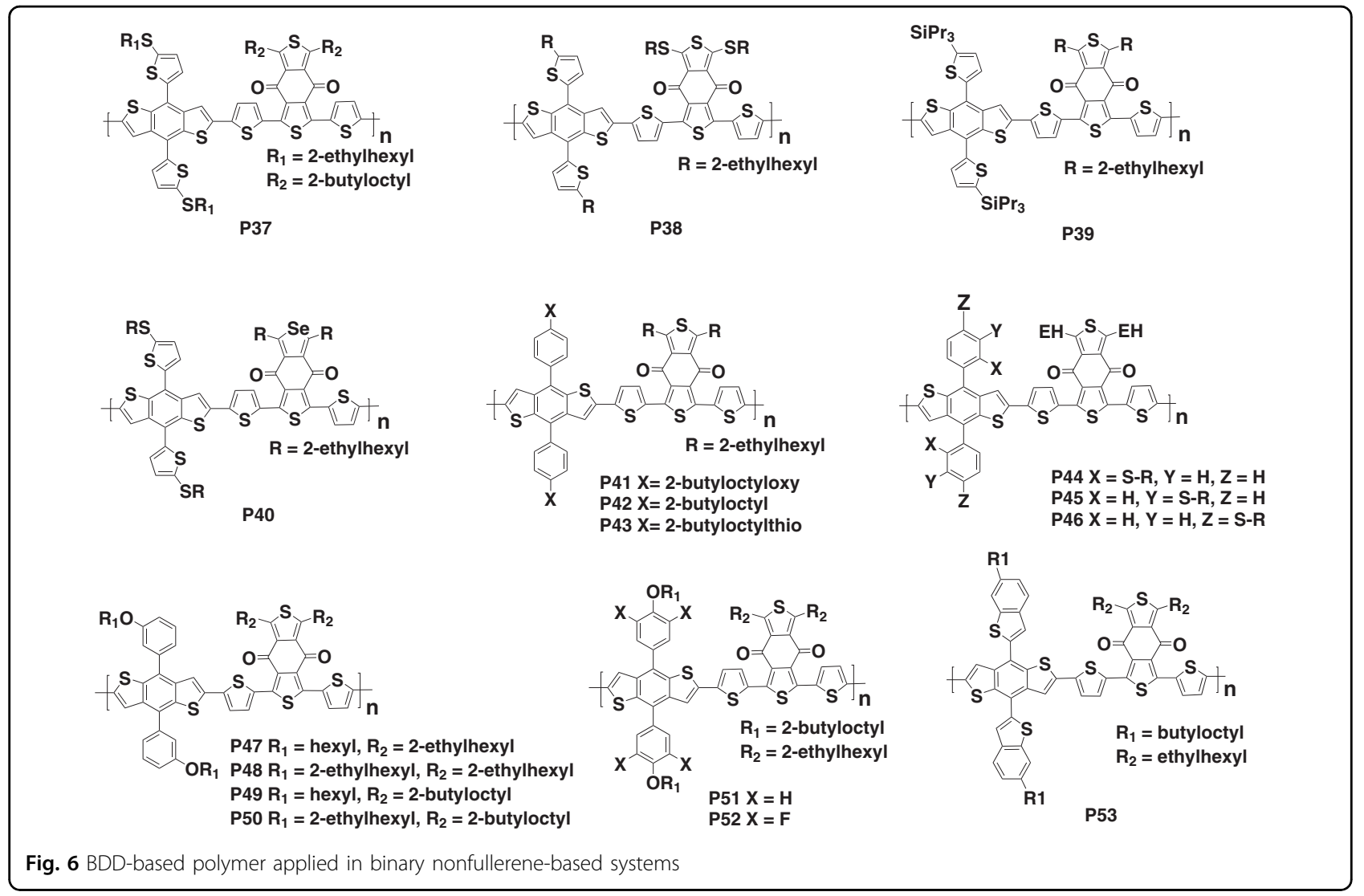

P34 and P35. This study provides additional design strategies for realizing low- and middle-bandgap BDDbased polymers.

\section{BDD-based polymer donors used in nonfullerene- acceptor-based OPVs}

In the last two decades, fullerene derivatives as pivotal components have been extensively explored to develop small molecule acceptors for OPVs. Despite the achievements of fullerene derivatives in laboratoryscale applications, the difficulty of tuning electronic energy levels, weak light absorption in visible light and poorer morphology stability hamper their further commercial applications. Therefore, the development of nonfullerene materials has drawn increasing attention in the study of OPVs due to their overwhelming features in terms of chemical structural flexibility, fabrication versatility, tunable absorption, energy level, crystallinity, etc ${ }^{34-36}$.

In some efficient nonfullerene solar cells, utilizing $\mathrm{BDT}$ as the D unit and BDD as the A unit to construct $\mathrm{D}-\mathrm{A}$ polymers could realize highly efficient PCEs over $14 \%$. The main contributions of the BDD-based polymer to the PCE originate from its complementary absorption, with a low-bandgap nonfullerene-acceptor, reasonable energy level matching with the acceptor, high crystalline property of thin films and reasonable phase separation for charge transport. Following these merits, some molecular design strategies involving variation of the conjugated side chains, functional groups and soluble alky chains on BDT and BDD units have predominated in recent years. For example, the P37 copolymer consisting of alkylthio side chains in BDT and BDD was reported by Huo and Sun et al. (Fig. 6) ${ }^{37}$. By adopting P37 as a donor and ITCPTC as a nonfullerene-acceptor, the blend exhibited a high PCE of $11.8 \%$ with a $V_{\text {oc }}$ of $0.95 \mathrm{~V}$, a $J_{\text {sc }}$ of $16.5 \mathrm{~mA} \mathrm{~cm}^{-2}$, and an FF of 0.751 . The $J_{\mathrm{sc}}$ was significantly improved due to their complementary absorption spectra to ensure full harvest of the incident light. The P38 copolymer was synthesized by using ethylhexylthio side chains to replace the alkyl side chain of its counterpart ${ }^{38,39}$. Unexpectedly, P38 exhibited poor crystallinity, low hole mobility and relatively low photovoltaic performance. Yang et al. designed a novel P39 copolymer via introducing a trialkylsilyl substituent into the BDT unit and proved that the acceptor excitation followed by hole transfer to the donor process was crucially important for more photocurrent generation ${ }^{40,41}$. Ultimately, the blend of P39:IDIC showed the best PCE of $12.01 \%$, 


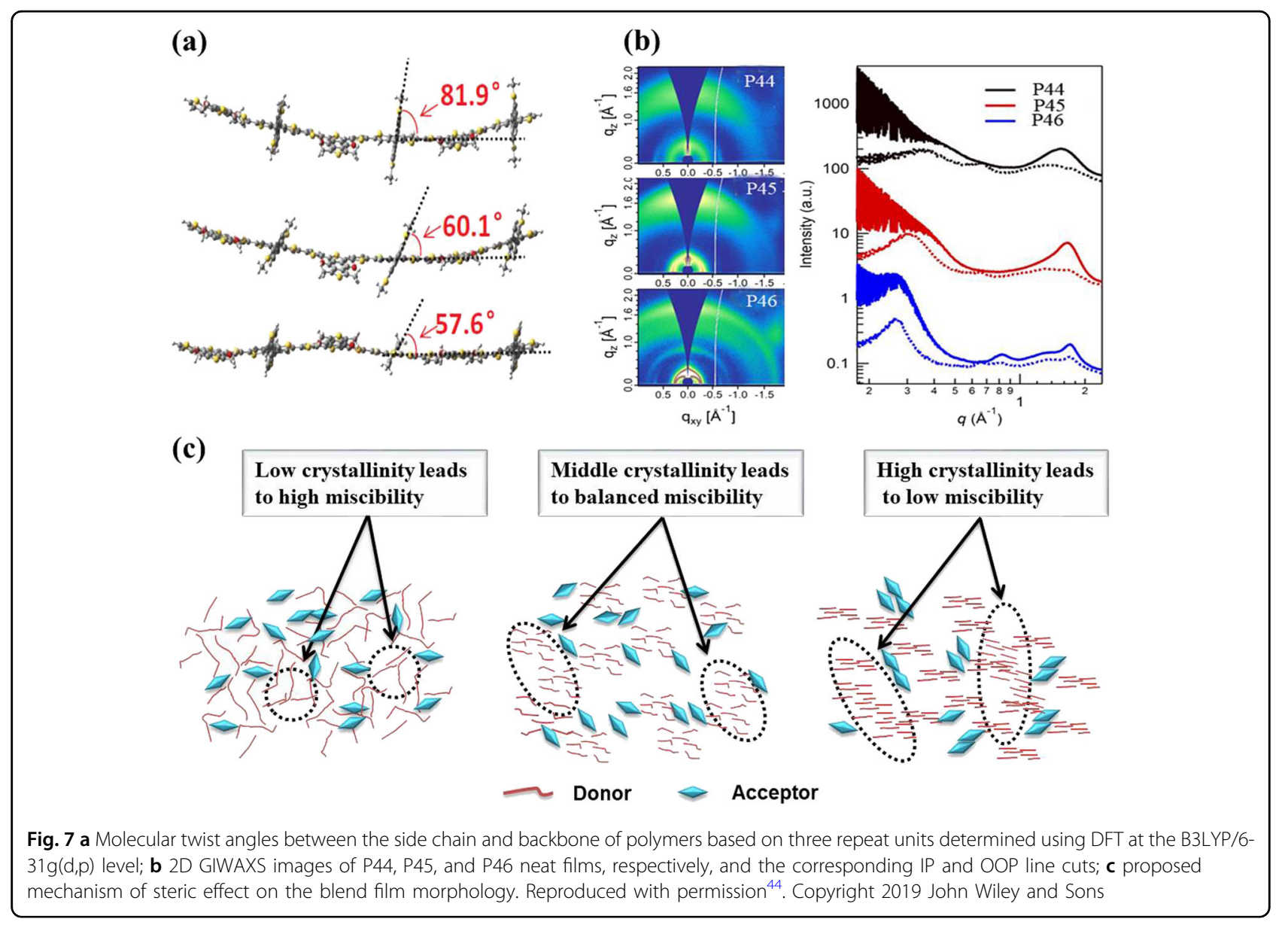

which was primarily attributed to the high $J_{\mathrm{sc}}$. This finding provides unique insight into efficient charge separation and transport for photocurrent generation mechanisms. The alternating BDT- and seleniumsubstituted BDD copolymer called $\mathbf{P 4 0}$ reported by Huo et al. exhibited a high PCE of $8.22 \%$ based on the perylene diimide (PDI) dimer acceptor, which coincided with the optimized blend films with a broad absorption range, balanced charge carrier mobility and reasonable morphology ${ }^{42}$.

In addition, a "linkage" between the conjugated building block and soluble alkyl side chains was revaluated by Huo and Sun et al. They designed three polymers (P41-P43) with alkoxyl, alkyl, and alkylthio side chains on the BDT unit ${ }^{43}$. P41 with alkoxyl chains and P43 with alkylthio chains showed significantly poor device performance compared with that of $\mathbf{P 4 2}$ with alkyl chains due to the pronounced broader fibril width caused by overaggregated behaviors of the alkoxyl and alkylthio chains compared with the alkyl chains and thus rendered incompatible phase separation. To finely tune the blend miscibility, Huo et al. designed a series of polymers P44-P46 by varying the alkylthiol side chain orientations ${ }^{44}$. It is interesting to note that the moderate steric effect of $\mathbf{P 4 5}$ possessed the highest PCE of $12.2 \%$ compared with P44 and P46 with side chains in orthoand para-positions, respectively, which is ascribed to the steric-effect-induced miscibility (SEIM). P45 with side chains in meta-positions promoted middle crystallinity and led to balanced miscibility and the best morphology (see Fig. 7). This method provides a feasible chemical tool for adjusting the blend miscibility. Guo et al. designed and prepared a class of structurally similar copolymers by incorporating meta-alkoxyphenylsubstituted BDT into the BDD unit to form steric effects on P47-P50 ${ }^{45}$. Among these copolymers, P47 with the shortest alkyl side chains showed the greatest photovoltaic performance, which was attributed to the strongest molecular aggregation and most attractive interactions in the $\mathbf{P 4 7}$ blend. However, other copolymers (P48, P49, and P50) with gradually increased alkyl chains led to aggregation, and the effective crystallinity was damaged. Therefore, for polymers with steric hindrances, it is important to maintain reasonable crystallinity by decreasing the alkyl chain length without sacrificing solubility. 

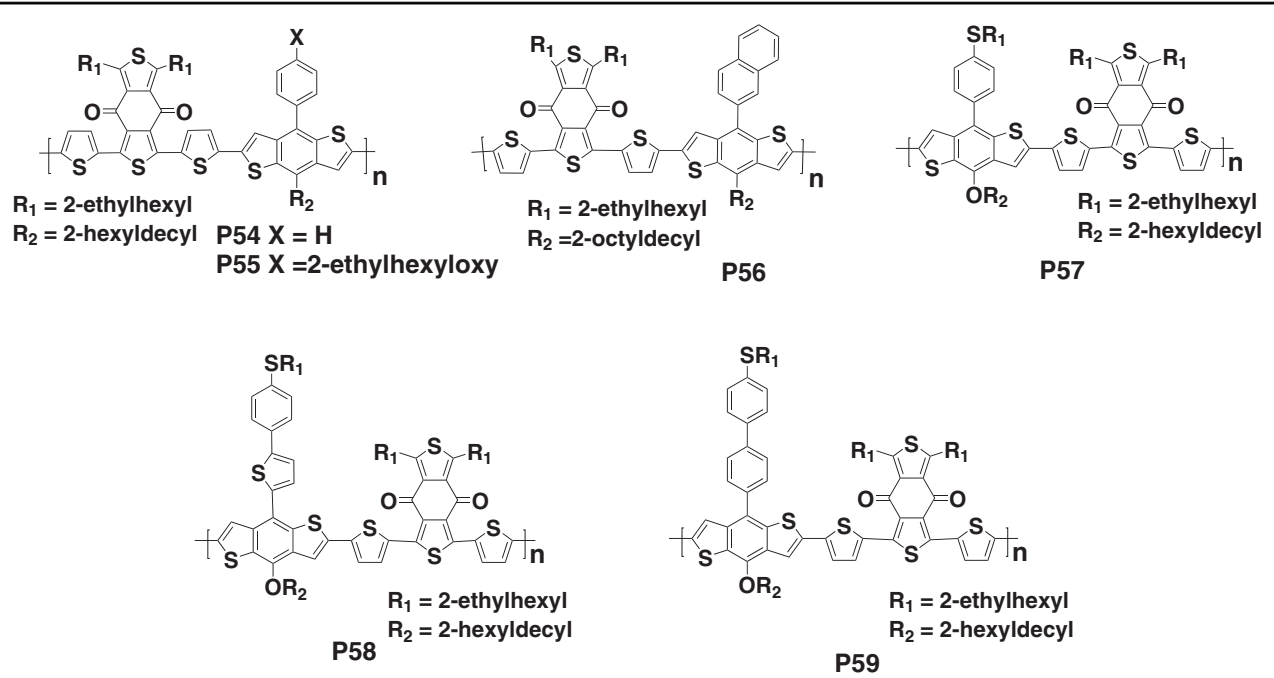

Fig. 8 BDD-based asymmetrical side chain polymer applied in binary nonfullerene-based systems

To further enhance main chain $\pi-\pi$ stacking and interchain interactions to facilitate charge transfer, side chain conjugation is strengthened in some cases. For example, fluorinated alkoxyphenyl side chains on the BDT unit were copolymerized with the BDD unit by Zhang et al. to obtain P52. For a clear comparison, P51 without a fluorinated counterpart was also prepared. The latter showed obviously inferior results (PCE of $6.2 \%$ with a $V_{\text {oc }}$ of $0.72 \mathrm{~V}$, a $J_{\mathrm{sc}}$ of $16.8 \mathrm{~mA} \mathrm{~cm}{ }^{-2}$ and an $\mathrm{FF}$ of $\left.51.2 \%\right)$ to those of P52 (PCE of $11.7 \%$ with a $V_{\text {oc }}$ of $0.89 \mathrm{~V}$, a $J_{\text {sc }}$ of $20.4 \mathrm{~mA} \mathrm{~cm}^{-2}$, and an FF of $\left.64.5 \%\right)^{46}$. The deeper HOMO level of P52 than that of P51 originated from the side chain fluorination, which contributed to a higher $V_{\mathrm{oc}}$. In addition, they found that fluorination on the side chain of P52 could suppress trap-assisted recombination and improve exciton dissociation to promote the improvement of $J_{\mathrm{sc}}$. Moreover, a higher coherence length (CL) of $P 52$ in the $\pi-\pi$ stacking direction was recorded, which was favorable for the charge transport, yielding a higher $J_{\mathrm{sc}}$ and FF. Hou et al. further extended conjugated side chains in the BDT- and BDD-based polymer P53 by replacing thienyl with larger-sized phenylthienyl units and longer soluble side chains ${ }^{47}$. The aim of enlarging its flexible side chains is to improve its solubility in nonhalogen solvents, and strengthening its conjugated side groups results in enhancement of its $\pi-\pi$ stacking effect in solid films. As a result, through this two-step optimization of the chemical structure, P53 exhibited a moderate aggregation effect in THF solution and strong $\pi-\pi$ stacking intensity and faceon originations in solid film, corresponding to a high PCE of $12.10 \%$, which is among the top values obtained by using nonchlorinated and non-aromatic main solvents.

Yang and coworkers have carried out a significant amount of work to design the asymmetric side chain structures of copolymers based on BDT- and BDDbased polymers (Fig. 8). The copolymers P54 and P55 were synthesized based on asymmetric BDT side chain units (with the bare phenyl or alkoxyl chain modified phenyl group as the substitution $)^{48}$. The PSCs of $\mathbf{P 5 5}$ exhibited a higher efficiency $(9.06 \%)$ than that of P54 (7.46\%) since the former possessed reinforced intermolecular interactions and a better miscibility with the acceptor by the alkoxyl chains attached onto the bare phenyl substitutions of the asymmetric BDT. It is preliminarily illustrated that the optimization of photovoltaic properties by the simple introduction of an asymmetric side chain geometry is an effective approach. To further investigate the influence of an asymmetric structure on the photovoltaic performance, the P56 copolymer was designed based on $\beta$-naphthalene asymmetric-modified BDT and BDD units $^{49}$. This copolymer showed an enhancement in solubility and a decrease in HOMO levels due to the large steric hindrance caused by the naphthalene group. Particularly, in nonfullerene-based PSCs, lower energy loss of the P56 blend was acquired, resulting in the coordination of $V_{\mathrm{oc}}$ and $J_{\mathrm{sc}}$, which synergistically improved the photovoltaic performance. The P57 polymer was synthesized by the same group via introducing alkoxybiphenyl as the substituted group into the BDT unit. As a result, P57 with an enhanced conjugated degree with more balanced charge transport rendered an efficient PCE. Moreover, they reported the creation of polymers P58 and P59 by inserting a benzene ring between the aryl and thioether in the asymmetric side chains as the substituted group into the BDT unit $^{50}$. Through the extended aryl side chains, the elongated nonfullerene-acceptor was stirred to repress 

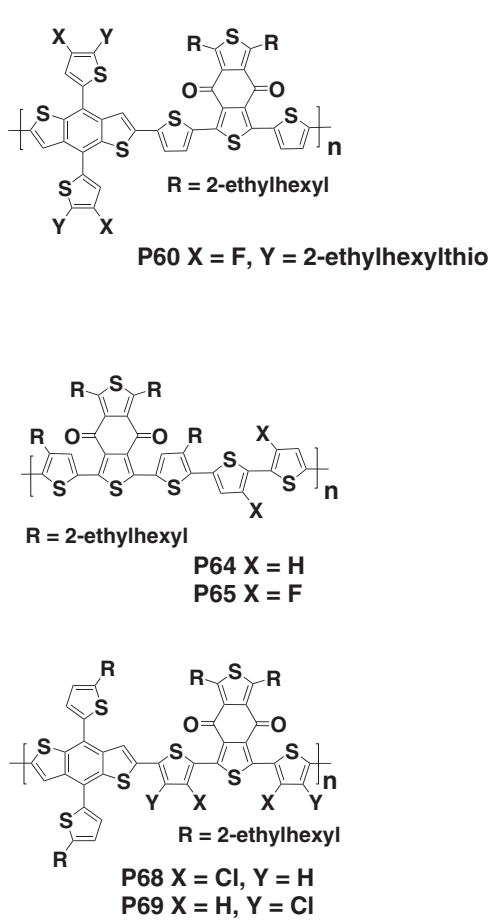
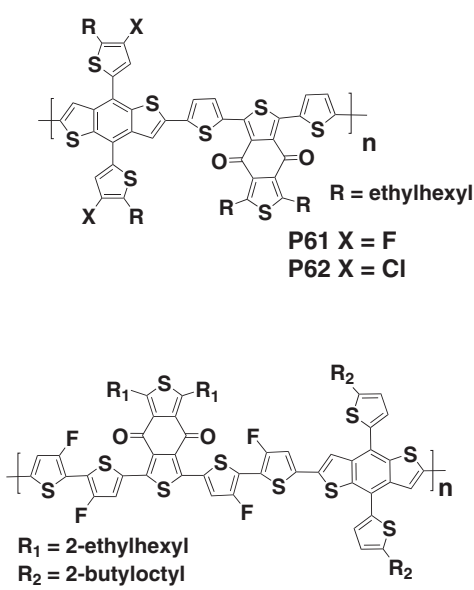

P66

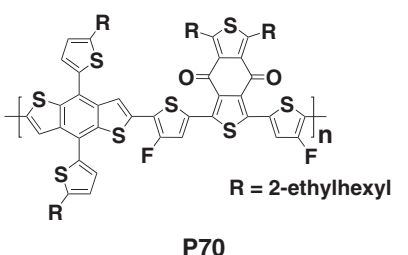

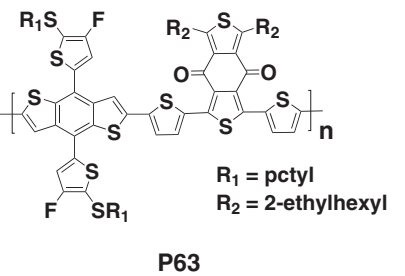
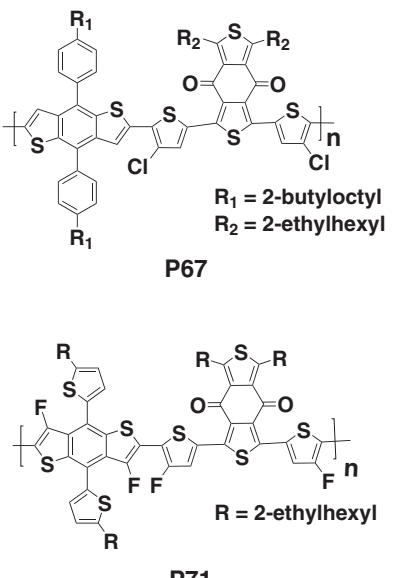

P71

Fig. 9 Halogen-group-affiliated polymers applied in binary nonfullerene-based systems

self-aggregation, and better miscibility was realized in the blend films, resulting in an optimized morphology and PCE. All these works represent an efficient strategy for controlling the morphology by a modified side chain and afford a promising PCE.

The halogen-group modifications (such as fluorination and chlorination) on the thienyl units have proved their potential in regulating molecular energy levels, enlarging the absorption ranges and enhancing the inter/intramolecular interactions in PSCs (Fig. 9). Following this strategy, Hou et al. synthesized a $\mathbf{P 6 0}$ copolymer based on fluorinated thiophene as the conjugated side group. For comparison, $\mathbf{P 3}$ was prepared without fluorination ${ }^{51}$. The results showed that the PCE of $\mathbf{P 6 0}$ (over 13\%) was higher than that of P3, which was ascribed to the broader optical absorption range and enhanced absorption coefficient yielded by the fluorinated thienyl side chains. Additionally, the same group fabricated two similar copolymers based on the bis-2-ethylhexyl thienyl-substituted BDT as the $\mathrm{D}$ unit and BDD as the A unit. The difference is that the thienyl side groups in $\mathbf{P 6 1}$ were fluorinated, while those of P62 were chlorinated ${ }^{52}$. Compared with P61, P62 showed pronounced advances with a relatively easier synthetic route and higher performances. From the device results, P61 showed a PCE of $13.2 \%$ with a $V_{\text {oc }}$ of $0.84 \mathrm{~V}$, a $J_{\mathrm{sc}}$ of $20.81 \mathrm{~mA} \mathrm{~cm}^{-2}$, and an FF of 0.76, and a P62-based device showed a $V_{\mathrm{oc}}$ of $0.86 \mathrm{~V}$, a $J_{\mathrm{sc}}$ of $21.80 \mathrm{~mA} \mathrm{~cm}^{-2}$, an
FF of 0.76, and an elegant PCE of 14.4\%. Later, Zou et al. developed a new acceptor of Y6 and made a blend of P61 and Y6, which delivered a very impressive high PCE of $15.7 \%$, and a certified efficiency of $14.9 \%$ was demonstrated $^{13}$. Therefore, the choice of a reasonable nonfullerene-based acceptor is crucial for achieving high performance. To further simplify the processing conditions, they adopted P63 as a donor by a solutionprocessed layer-by-layer (LBL) approach ${ }^{53}$. The PCE of $13.0 \%$ for the LBL device was obviously enhanced compared to that of the one-step-processed device (11.8\%). The exact reasons for the enhancement were the decreased carrier traps and the reduced bimolecular recombination. Thus, the LBL method showed a greater potential for device processing.

In addition to the various halogen-group modifications on thienyl side chains attached to the $\mathrm{D}$ unit, recent research has shown that fluoro- and chloro-substituted $\pi$ bridges, even main chain blocks, play a vital role in adjusting energy levels, optimizing crystallinity and improving photovoltaic performance. For example, the copolymers P64 and P65 were designed by Hou et al. for comparison in a bithiophene $\pi$-bridge and its fluorinated analogue $^{54}$. The results showed that P65 with a fluorinated $\pi$-bridge not only decreased the HOMO levels but also pronouncedly improved the $\pi-\pi$ stacking degree in the solid film. Correspondingly, the higher photovoltaic 
property of P65 over that of P64 acted as a more ideal candidate for making front cells in double-junction tandem PSCs. In addition, several cases reported similar molecular modification strategies for BDD-based $\pi$ bridges. The P66 copolymer based on the more fluorinesubstituted bithiophene $\pi$-bridge was successfully synthesized and exhibited distinguished photovoltaic properties $^{55}$. Sun et al. showed the P67 copolymer with a chlorinated thienyl $\pi$-bridge, further proving the positive effect of halogen-group modifications on the thienyl $\pi$ bridge and corresponding device performance ${ }^{56}$. At the same time, the relationship between the halogen-group origination in the $\pi$-bridge and the photovoltaic performance was also investigated. Hou et al. synthesized two copolymers (P68 and P69) with different orientations for the chlorinated thienyl $\pi$-bridge ${ }^{57}$. $\mathbf{P 6 8}$ showed a particularly low PCE of $0.18 \%$, which was due to extremely weak intra/intermolecular charge transport owing to the highly twisted conjugated backbone of $\mathbf{P 6 8}$ and the corresponding poor morphology. By contrast, P69 exhibited an outstanding PCE of $12.33 \%$, with a $J_{\text {sc }}$ of $20.6 \mathrm{~mA} \mathrm{~cm}^{-2}$ and an FF of $71.09 \%$ owing to the well-balanced charge carrier mobilities and good phase-separation morphology of the blend film. This work suggested that chlorinated thienyl orientations need to be considered in designing efficient BDD-based donor polymers. Heeney et al. synthesized a new P70 copolymer with a fluorinated $\pi$-bridge and also exhibited a preeminent PCE of $13.2 \%$. The introduction of a fluorinated thienyl $\pi$-bridge was responsible for the reduced energy loss of $0.6 \mathrm{eV}$ and a high $J_{\text {sc }}$ of $19.6 \mathrm{~mA} \mathrm{~cm}{ }^{-2}$ in P70 blends ${ }^{58}$. Notably, overfluorination will generate adverse influences. Nelson et al. synthesized the P71 copolymer by introducing four $\mathrm{F}$ atoms ${ }^{59}$. The device of P71 showed a large $V_{\mathrm{oc}}$
$(>0.85 \mathrm{~V})$; however, overfluorination led to a poorer $\mathrm{FF}$ and $J_{\mathrm{sc}}$.

To facilitate better comprehension of the structureproperty relationships in BDD-based polymer donors for application in nonfullerene OPVs, several research groups have focused on their conformation varieties. Chen et al. designed a set of experiments to explore the relationship between photovoltaic properties and molecular conformation on the main backbone. Through tuning $\pi-$ bridges (without and with thiophene or 3-hexylthieno[3,2b]thiophene) between the BDT and BDD units, these polymers with different conformation tendencies for P72 (zigzag), P73 (linear), and P74 (zigzag) exhibited different aggregation states ${ }^{60}$. Since the alkyl side chains yielded a steric twist angle, P74 had a more twisted backbone than that of P73 and led to a change from linear to zigzag conformation. As a result, the extended zigzag conformation of P74 showed moderate aggregation behavior, coupled with a more optimized blend morphology and a superior PCE. Yang and Sun et al. designed another series of polymers (P75, P76, and P77) by replacing one sulfur atom in the BDT core with one oxygen atom ${ }^{61}$ (Fig. 10). The synergistic effect of both side chain and backbone conformational engineering resulted in an elegant performance.

The fused polycyclic group possesses a large planar conjugated structure and facile $\pi-\pi$ stacking conformation, which is beneficial for charge transfer in a blend film and crystallinity. Lu and Wei et al. reported a series of alternating naphthodithiophene (NDT) and BDD copolymers P78, P79, and P80 created by modifying the NDT side chain and $\pi$-bridges ${ }^{62}$. Among the three copolymers, P78 exhibited a best PCE of $9.72 \%$ due to its face-on orientation and good crystallinity, which enhance the

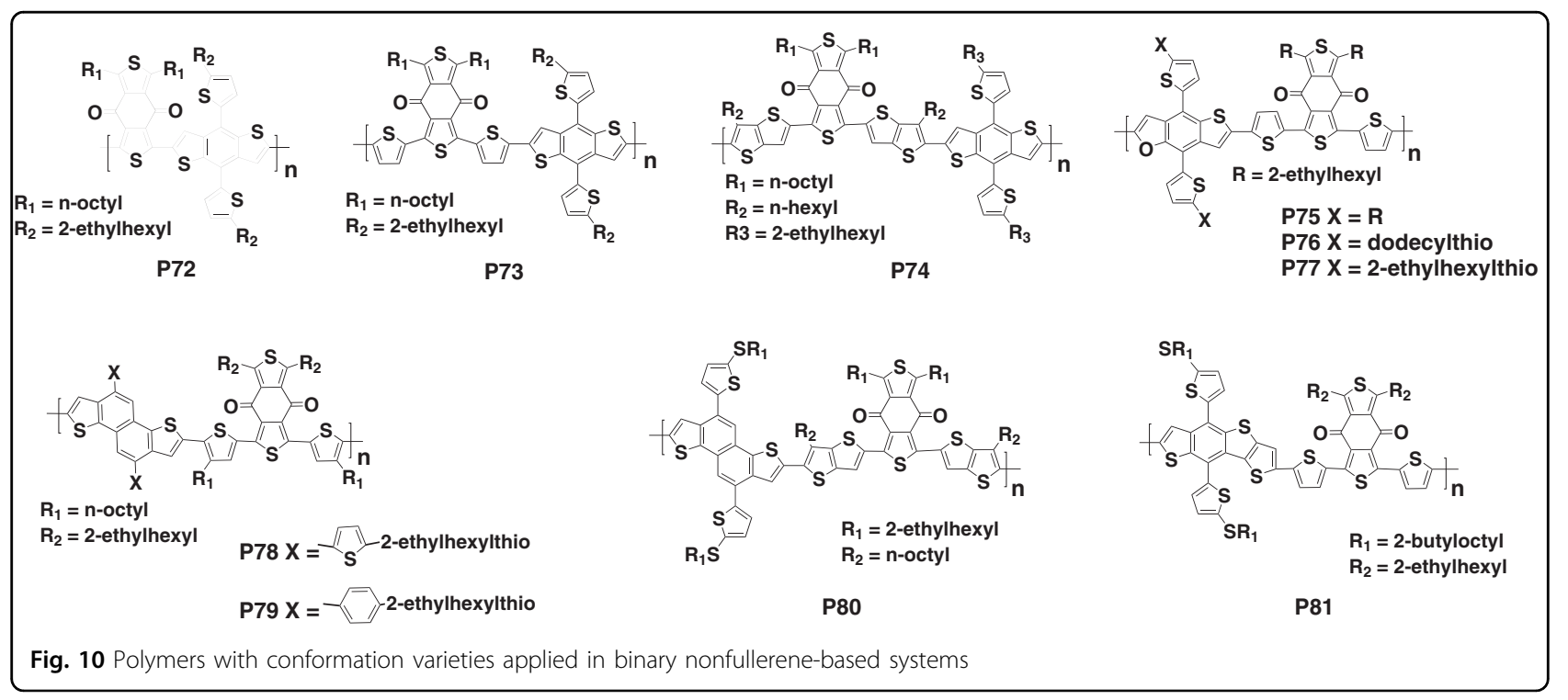


Table 2 Polymer characteristics and the corresponding device performances of nonfullerene-based binary OPVs

\begin{tabular}{|c|c|c|c|c|c|c|c|c|c|}
\hline Donor & $E_{\mathrm{g}}^{\mathrm{opt}}(\mathrm{eV})$ & HOMO/LUMO (eV) & Acceptor & $\mu_{\mathrm{h}}\left(\mathrm{cm}^{2} \cdot \mathrm{V}^{-1} \cdot \mathrm{s}^{-1}\right)$ & $J_{\mathrm{sc}}\left(\mathrm{mA} \mathrm{cm}{ }^{-2}\right)$ & $V_{\text {oc }}(\mathrm{V})$ & FF (\%) & PCE (\%) & ref \\
\hline P37 & 1.58 & $-5.62 /-3.96$ & ITCPTC & $1.71 \times 10^{-3}$ & 16.50 & 0.95 & 75.1 & 11.80 & 37 \\
\hline P38 & 1.85 & & ITIC & $4.7 \times 10^{-5}$ & 10.45 & 0.96 & 50.0 & 5.00 & 38 \\
\hline P39 & 1.81 & $-5.14 /-3.33$ & IDIC & & 17.00 & 0.939 & 75.3 & 12.01 & 40 \\
\hline P40 & & $-5.34 /-3.53$ & SdiPBI-S & $2.6 \times 10^{-3}$ & 12.80 & 0.91 & 68.8 & 8.22 & 42 \\
\hline P41 & 1.82 & $-5.30 /-3.30$ & ITCPTC & & 12.60 & 0.91 & 64.0 & 7.40 & 43 \\
\hline P42 & 1.84 & $-5.43 /-3.36$ & ITCPTC & & 17.00 & 0.94 & 78.0 & 12.70 & 43 \\
\hline P43 & 1.83 & $-5.45 /-3.37$ & ITCPTC & & 15.20 & 0.94 & 66.7 & 9.60 & 43 \\
\hline P44 & 1.89 & $-5.52 /-3.37$ & ITCPTC & $5.38 \times 10^{-5}$ & 8.43 & 1.00 & 36.6 & 3.09 & 44 \\
\hline P45 & 1.88 & $-5.48 /-3.42$ & ITCPTC & $2.28 \times 10^{-4}$ & 18.11 & 0.96 & 70.2 & 12.22 & 44 \\
\hline P46 & 1.84 & $-5.41 /-3.49$ & ITCPTC & $1.15 \times 10^{-4}$ & 15.76 & 0.91 & 65.2 & 9.36 & 44 \\
\hline P47 & 1.85 & & IDIC & $3.07 \times 10^{-4}$ & 19.50 & 0.87 & 72.4 & 12.30 & 45 \\
\hline P48 & 1.90 & & $\mathrm{IDIC}$ & $1.89 \times 10^{-4}$ & 19.00 & 0.92 & 62.8 & 11.00 & 45 \\
\hline P49 & 1.92 & & IDIC & $1.88 \times 10^{-4}$ & 17.00 & 0.92 & 66.4 & 10.40 & 45 \\
\hline P50 & 1.92 & & $\mathrm{IDIC}$ & $1.62 \times 10^{-4}$ & 16.40 & 0.93 & 58.7 & 8.90 & 45 \\
\hline P51 & 1.81 & $-5.38 /-3.57$ & $\mathrm{IT}-4 \mathrm{~F}$ & & 16.80 & 0.72 & 51.2 & 6.20 & 46 \\
\hline P52 & 1.86 & $-5.50 /-3.64$ & $\mathrm{IT}-4 \mathrm{~F}$ & & 20.40 & 0.89 & 64.5 & 11.70 & 46 \\
\hline P53 & & $-5.38 /$ & IT-M & $7.7 \times 10^{-5}$ & 17.97 & 0.96 & 70.0 & 12.10 & 47 \\
\hline P54 & 1.86 & $-5.31 /-3.45$ & ITIC & $2.10 \times 10^{-4}$ & 13.81 & 0.90 & 59.9 & 7.46 & 48 \\
\hline P55 & 1.84 & $-5.29 /-3.45$ & ITIC & $1.95 \times 10^{-4}$ & 16.70 & 0.90 & 60.3 & 9.06 & 48 \\
\hline P56 & 1.83 & $-5.41 /-3.58$ & ITIC & $2.91 \times 10^{-4}$ & 16.81 & 0.94 & 66.3 & 10.50 & 49 \\
\hline P57 & 1.75 & $-5.37 /-3.52$ & ITIC & $1.96 \times 10^{-5}$ & 14.65 & 0.92 & 53.8 & 7.23 & 50 \\
\hline P58 & 1.75 & $-5.36 /-3.51$ & ITIC & $3.12 \times 10^{-4}$ & 16.95 & 0.91 & 68.2 & 10.51 & 50 \\
\hline P59 & 1.74 & $-5.39 /-3.54$ & ITIC & $3.27 \times 10^{-4}$ & 17.07 & 0.90 & 65.9 & 10.07 & 50 \\
\hline P60 & 1.80 & $-5.28 /-3.48$ & ITIC & $2.10 \times 10^{-4}$ & 20.88 & 0.88 & 71.3 & 13.10 & 51 \\
\hline P61 & & $-5.47 /$ & $\mathrm{IT}-4 \mathrm{~F}$ & $2.97 \times 10^{-4}$ & 20.81 & 0.84 & 76.0 & 13.20 & 52 \\
\hline P61 & 1.81 & $-5.56 /-3.50$ & Y6 & & 25.20 & 0.82 & 76.1 & 15.70 & 13 \\
\hline P62 & & $-5.51 /$ & $\mathrm{IT}-4 \mathrm{~F}$ & $2.13 \times 10^{-4}$ & 21.80 & 0.86 & 77.0 & 14.40 & 52 \\
\hline P63 & & $-5.44 /-3.56$ & $\mathrm{IT}-4 \mathrm{~F}$ & $6.64 \times 10^{-5}$ & 20.30 & 0.90 & 71.0 & 13.00 & 53 \\
\hline P64 & & $-5.39 /$ & ITIC & $2.9 \times 10^{-3}$ & 2.04 & 0.88 & 68.0 & 6.53 & 54 \\
\hline P65 & & $-5.30 /$ & ITIC & $3.5 \times 10^{-3}$ & 15.04 & 0.94 & 61.0 & 8.69 & 54 \\
\hline P66 & 1.79 & $-5.48 /-3.58$ & ITIC & $1.41 \times 10^{-4}$ & 15.92 & 0.94 & 68.3 & 10.26 & 55 \\
\hline P67 & 1.85 & $-5.59 /-3.53$ & $\mathrm{IT}-4 \mathrm{~F}$ & $1.34 \times 10^{-3}$ & 19.74 & 0.85 & 76.0 & 12.70 & 56 \\
\hline P68 & 2.11 & $-5.54 /-3.27$ & $\mathrm{IT}-4 \mathrm{~F}$ & & 0.88 & 0.88 & 23.74 & 0.18 & 57 \\
\hline P69 & 1.78 & $-5.48 /-3.47$ & $\mathrm{IT}-4 \mathrm{~F}$ & $2.57 \times 10^{-5}$ & 20.60 & 0.84 & 71.09 & 12.33 & 57 \\
\hline P70 & 1.77 & $-5.47 /-3.46$ & C8-ITIC & $4.4 \times 10^{-4}$ & 19.60 & 0.94 & 72.0 & 13.20 & 58 \\
\hline P71 & 1.83 & $-5.63 /-3.52$ & $\mathrm{IT}-4 \mathrm{~F}$ & & 18.40 & 0.93 & 62.0 & 9.90 & 59 \\
\hline P72 & 1.87 & $-5.46 /-3.59$ & ITIC & $2.18 \times 10^{-6}$ & 5.77 & 0.95 & 39.0 & 2.13 & 60 \\
\hline P73 & 1.77 & $-5.35 /-3.58$ & ITIC & $1.17 \times 10^{-5}$ & 16.33 & 0.84 & 64.0 & 9.00 & 60 \\
\hline P74 & 1.83 & $-5.37 /-3.54$ & ITIC & $2.83 \times 10^{-5}$ & 15.52 & 0.88 & 68.0 & 9.21 & 60 \\
\hline
\end{tabular}


Table 2 continued

\begin{tabular}{|c|c|c|c|c|c|c|c|c|c|}
\hline Donor & $E_{\mathrm{g}}^{\mathrm{opt}}(\mathrm{eV})$ & HOMO/LUMO (eV) & Acceptor & $\mu_{\mathrm{h}}\left(\mathrm{cm}^{2} \cdot \mathrm{v}^{-1} \cdot \mathrm{s}^{-1}\right)$ & $J_{s c}\left(m A c m^{-2}\right)$ & $V_{\text {oc }}(\mathrm{V})$ & FF (\%) & PCE (\%) & ref \\
\hline P75 & 1.80 & $-5.26 /-3.46$ & ITIC & $2.21 \times 10^{-4}$ & 17.76 & 0.89 & 70.16 & 11.13 & 61 \\
\hline P76 & 1.75 & $-5.31 /-3.56$ & ITIC & $1.08 \times 10^{-4}$ & 17.24 & 0.93 & 66.10 & 10.57 & 61 \\
\hline P77 & 1.75 & $-5.32 /-3.57$ & ITIC & $0.95 \times 10^{-4}$ & 17.06 & 0.94 & 64.1 & 10.23 & 61 \\
\hline P78 & 1.92 & $-5.24 /-3.46$ & IDIC & $2.46 \times 10^{-4}$ & 15.65 & 0.87 & 71.05 & 9.72 & 62 \\
\hline P79 & 1.94 & $-5.34 /-3.46$ & IDIC & $7.61 \times 10^{-5}$ & 10.63 & 0.92 & 46.9 & 4.62 & 62 \\
\hline P80 & 1.96 & $-5.29 /-3.40$ & IDIC & $1.29 \times 10^{-4}$ & 15.15 & 0.8901 & 59.65 & 8.20 & 62 \\
\hline P81 & 1.78 & $-5.46 /-3.72$ & ITIC & $1.16 \times 10^{-4}$ & 17.32 & 0.98 & 66.5 & 11.39 & 63 \\
\hline
\end{tabular}

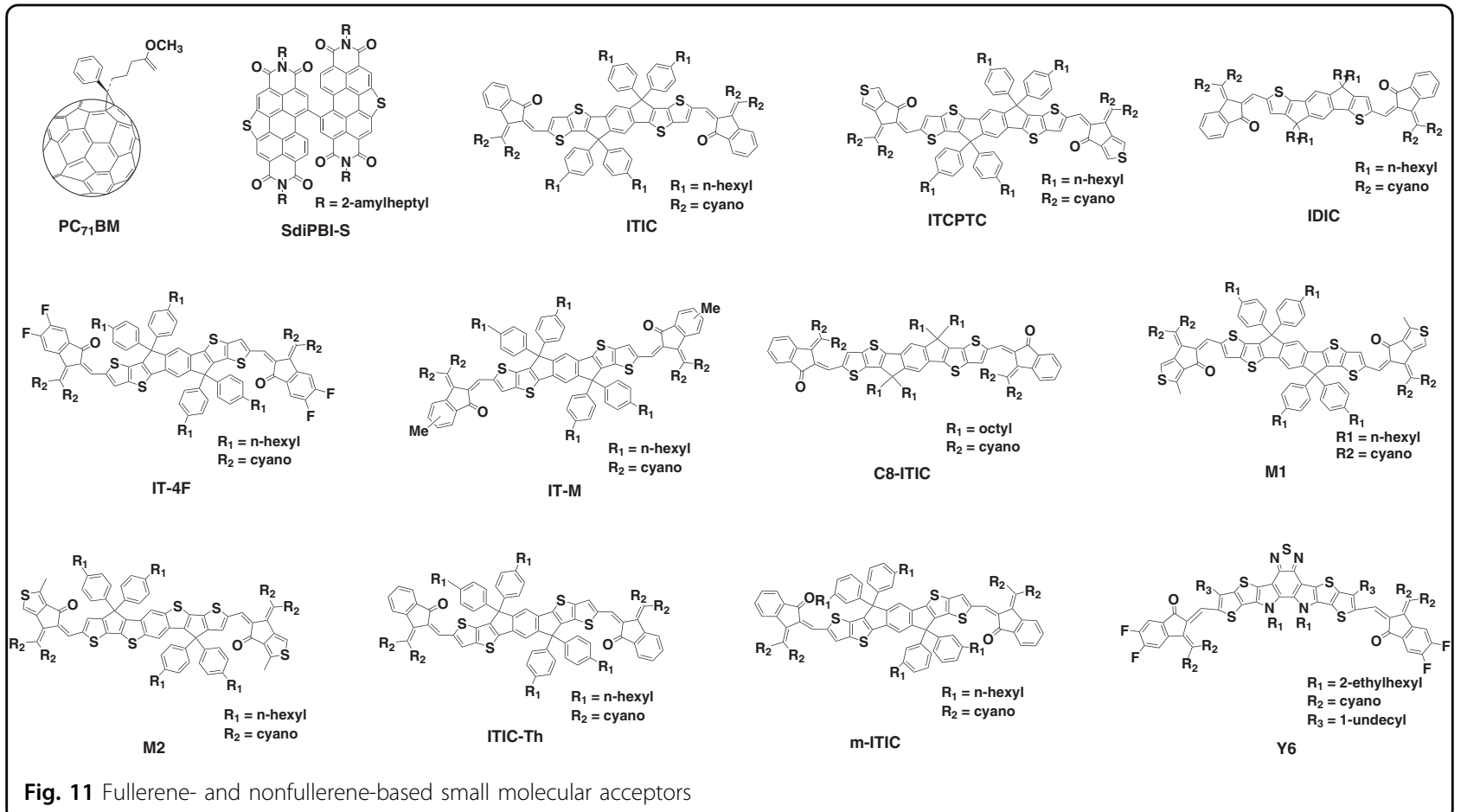

charge transport in the blending films. Unexpectedly, P79 showed a weak PCE of $4.62 \%$ because the phenyl side chain of the naphthodithiophene unit had a larger conformational torsion and thus resulted in suppression of the planarity, reducing the $J_{\mathrm{sc}}$ and FF values. Yang et al. designed a new asymmetrical backbone polymer called P81 containing thienobenzodithiophene (TBD) and BDD units $^{63}$. The asymmetrical TBD block could promote the photovoltaic performance by modulating molecular aggregation and crystallinity, and the outstanding photovoltaic devices of P81 achieved a PCE of $11.39 \%$ without any post-treatment. Table 2 summarizes the characteristics and the corresponding device performances of the nonfullerene-based binary OPVs.

\section{BDD-based polymer donors used in ternary OPVS}

Except for two components (donor and acceptor) as optical-active materials, the ternary strategy in OPVs has been considered a very promising candidate for enhancing the photovoltaic performance of polymer solar cells. This strategy with three different optical-active components (donor, acceptor, and a third component) not only can enhance photon harvesting by selecting the third component with complementary absorption spectra compared with the binary system but also can well optimize the morphology of active layers by incorporating the appropriate third component. Many reports also presented different working mechanisms of ternary OSCs, such as charge transfer, energy transfer, the alloy-like model, and 


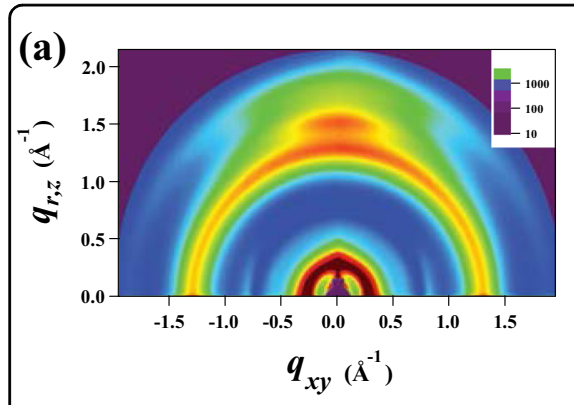

(d)

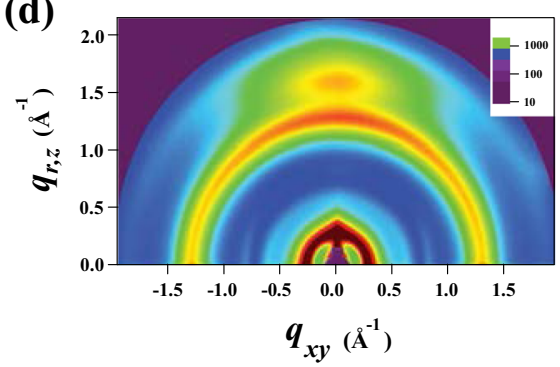

(f)

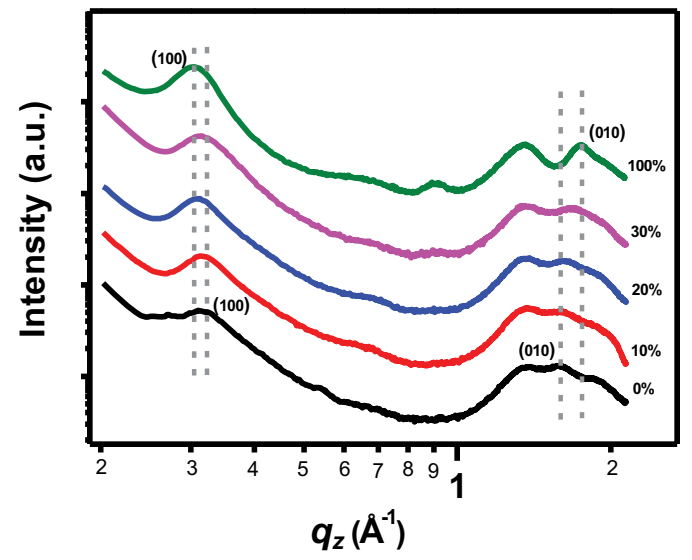

(b)
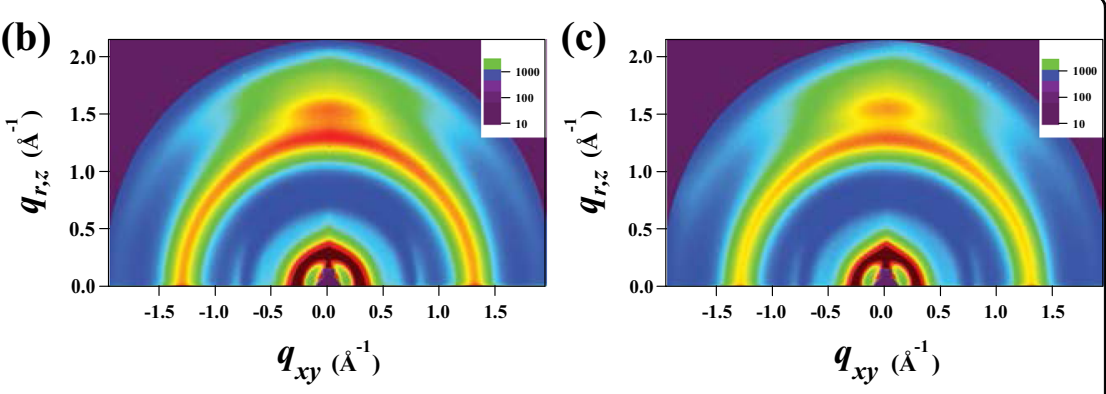

(e)

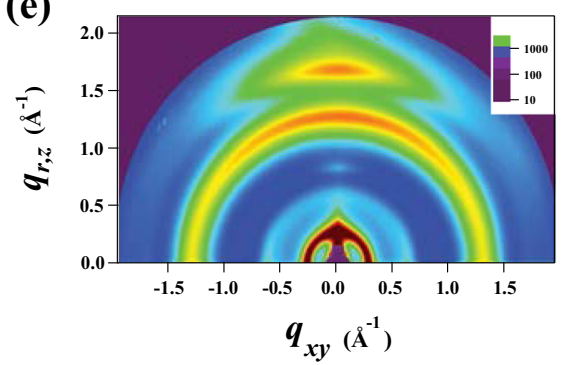

(g)

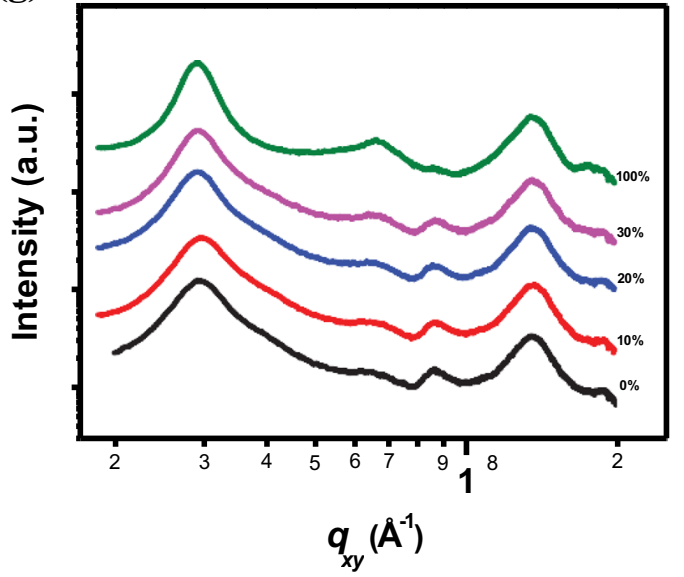

Fig. 12 2D GIWAXS patterns of ternary blend films with different weight ratios of P6: a 0\%, b 10\%, c 20\%, d 30\%, and e $100 \%$. f Out-of-plane and $\mathbf{g}$ in-plane line cuts from 2D GIWAXS patterns. Reproduced with permission ${ }^{67}$. Copyright 2016 John Wiley and Sons

the parallel-linkage model, etc ${ }^{64}$. For example, Zhang et al. developed efficient alloy-like ternary nonfullerene OPVs by utilizing $\mathbf{P 4 2}$ as a donor and two compatible nonfullerene small molecules of $\mathbf{M 1}$ and $\mathbf{M} 2$ as acceptors $^{65}$. The ternary OPVs with $30 \mathrm{wt} \% \mathbf{M} 2$ exhibited a higher PCE of $12.55 \%$, which was higher than that of the binary OPVs containing a single acceptor. In further analysis, they found that the improvement in electron transport played an important role in realizing high $J_{\mathrm{sc}}$ and FF because the two acceptors had similar LUMO energy levels. Sun and Huo et al. designed highly efficient alloy-like ternary OPVs by using a wide-bandgap polymer (P6) and a low-bandgap polymer of PTB7-Th as two donors and $\mathrm{PC}_{71} \mathrm{BM}$ as one acceptor (Fig. 11) ${ }^{66-68}$. From the 2D GIWAXS (Fig. 12), only one lamellar diffraction peak was observed for all ternary blends, indicating that P6 is well mixed with PTB7-Th polymer chains in the ternary blend. Increased P6 content of $\sim 20 \%$ in the ternary blend system leads to a balanced lamellar distance and $\pi-\pi$ stacking distance. Subsequently, an optimized morphology of the increased crystallinity and face-on orientation of the mixed polymers leads to high performance. This work suggested that two donors could work together as alloys in the ternary blends and did not lead to stronger bimolecular recombination and thus did not reduce charge carrier transport in the ternary blend films.

\section{BDD-based ternary polymer donors}

In addition to these practical physical ternary strategies, the exploitation of chemical ternary tools as an alternative 


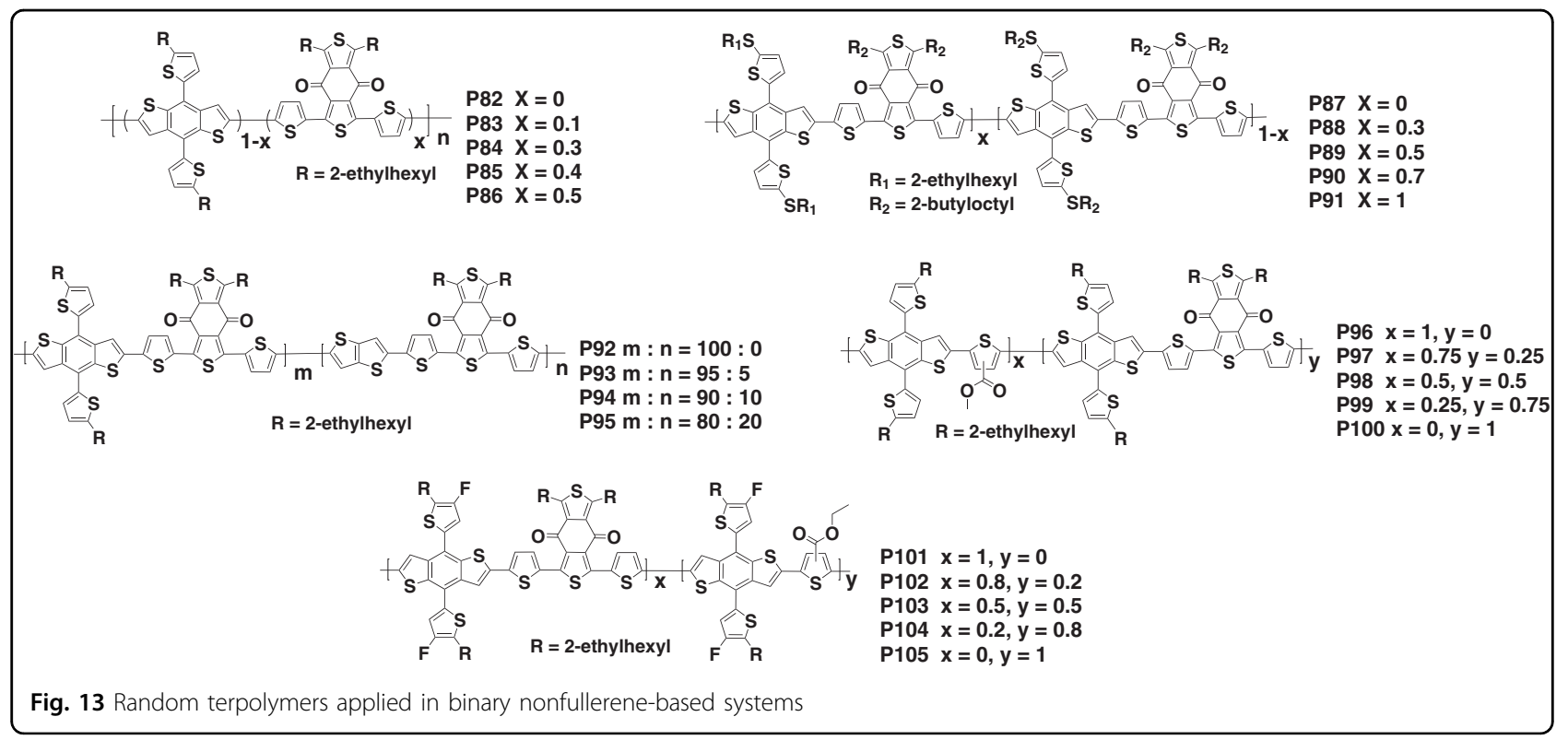

strategy to control the blend morphology is showing advantages in molecular-scaled fine control. The random copolymerization of three components in one main backbone has emerged as a simple and promising ternary tool for boosting the photovoltaic performance and finely tune the morphology, charge transport ability, electronic energy levels, and so on (Fig. 13). Sun and coworkers synthesized D-A alternating copolymers P82-P86 in which alternating BDT units and BDD units were employed $^{69}$. As a result, the random copolymer P84 with a 30\% BDD content exhibited the highest efficiency of $11.14 \%$ without any additional treatment among these copolymers, which is closely related to the percolating network structure caused by the large mixed phase in the P84 blend and resulted in efficient charge transport. Huo and Sun et al. reported the influence of subtle side chain engineering on the optical and electronic properties of random copolymers. They synthesized several copolymers (P87-P91) in which the subtle side chain regioregularity is used to finely tune the optical, electronic, and morphological properties ${ }^{70}$. $\mathbf{8 8 9}$ outperformed the other copolymers, with better mixing in fullerene or nonfullerene blends, and achieved high PCEs of 10.1 and $12.1 \%$, respectively. The results showed the importance of the subtle side chain engineering of random terpolymers when gradually tuning the crystal packing and optimizing the morphology. Yang developed a series of random copolymers P92-P95 based on donor polymer PBDB-T by introducing BDT and thieno $[3,2-b]$ thiophene (TT) as two D units and BDD as an A unit $^{71}$. The research showed that the TT content fraction over $10 \%$ in the polymer backbone would decrease the aggregation of copolymers, and with a further increase in TT content, the random copolymer significantly enhanced its $J_{\mathrm{sc}}$ up to $17.53 \mathrm{~mA} \mathrm{~cm}{ }^{-2}$, which was the main contributor to the high PCE value.

A similar tercopolymerization method has also been adopted by other research groups. Choi designed and synthesized a set of random terpolymers P96-P100 by introducing the BDT unit as the donor, methyl-3thiophenecarboxylate (3MT) as the weak electronaccepting unit, and BDD as a strong electron-accepting unit $^{72}$. Compared with the binary copolymer-based PSCs (P96:ITIC and P100:ITIC), the terpolymer-based PSCs of P98:ITIC exhibited a higher PCE of $10.26 \%$ with a high $V_{\text {oc }}$ of $0.94 \mathrm{~V}$ and $J_{\text {sc }}$ of $17.18 \mathrm{~mA} \mathrm{~cm}^{-2}$. The outstanding photovoltaic performance can be attributed to the more balanced charge transport, the existing face-on orientation behavior and the proper crystallinity in the blend film. On the other hand, the ITIC molecules could be easily intercalated into the polymer chain network because random terpolymers impeded the regular ordering, thus decreasing the degree of crystallization of the polymer backbones. Hou et al. also reported a series of similar random copolymers P101-P105 by inserting an ester-group-substituted thiophene unit into the BDT and $\mathrm{BDD}$ units $^{73}$. As the ratio of 3-thiophenecarboxylate increased, the dihedral angle caused by 3thiophenecarboxylate gradually increased along the main chain backbone, resulting in decreased polymeric crystallinity. Compared with binary OSC (P105:IT-4F), the P101:IT-4F-based device yields a maximum PCE of $15.1 \%$, with a $V_{\text {oc }}$ of $0.899 \mathrm{~V}$, a $J_{\text {sc }}$ of $21.5 \mathrm{~mA} \mathrm{~cm}^{-2}$, and an FF of 0.78. From all these cases, it is concluded that the overaggregation issue in high crystallite polymer could be efficiently overcome by the simple, efficient method of random copolymerization, which will enable a more 
Table 3 Polymer characteristics and the corresponding device performances of ternary and terpolymer-based OPVs

\begin{tabular}{|c|c|c|c|c|c|c|c|c|c|}
\hline Donor & $E_{\mathrm{g}}^{\mathrm{opt}}(\mathrm{eV})$ & HOMO/LUMO (eV) & Acceptor & $\mu_{\mathrm{h}}\left(\mathrm{cm}^{2} \cdot \mathrm{v}^{-1} \cdot \mathrm{s}^{-1}\right)$ & $J_{s c}\left(m A c m^{-2}\right)$ & $V_{\text {oc }}(\mathrm{V})$ & $\mathrm{FF}(\%)$ & PCE (\%) & ref \\
\hline $\mathrm{P} 42$ & 1.84 & $-5.43 /-3.36$ & M1:M2 & $3.2 \times 10^{-5}$ & 17.84 & 0.93 & 75.2 & 12.55 & 65 \\
\hline P6 & 1.85 & $-5.36 /-3.43$ & ITIC-Th: $P C_{71} B M$ & $2.17 \times 10^{-3}$ & 15.54 & 0.93 & 70.5 & 10.48 & 66 \\
\hline P82 & 1.82 & $-5.36 /$ & ITIC & $6.89 \times 10^{-5}$ & 16.92 & 0.90 & 57.56 & 8.81 & 69 \\
\hline P83 & 1.83 & $-5.41 /$ & ITIC & $2.10 \times 10^{-4}$ & 17.56 & 0.94 & 66.9 & 11.08 & 69 \\
\hline P84 & 1.83 & $-5.45 /$ & ITIC & $2.94 \times 10^{-4}$ & 18.38 & 0.93 & 65.0 & 11.14 & 69 \\
\hline P85 & 1.89 & $-5.49 /$ & ITIC & $3.43 \times 10^{-5}$ & 16.25 & 0.91 & 49.9 & 7.44 & 69 \\
\hline P86 & 2.16 & $-5.51 /$ & & & & & & & 69 \\
\hline P87 & & $-5.40 /-3.54$ & $\mathrm{PC}_{71} \mathrm{BM} / \mathrm{ITCPT}$ & $1.2 \times 10^{-3}$ & $12.60 / 15.30$ & $0.92 / 0.91$ & $76.0 / 72.0$ & $8.90 / 10.30$ & 70 \\
\hline P88 & & $-5.42 /-3.56$ & $\mathrm{PC}_{71} \mathrm{BM} / \mathrm{ITCPT}$ & & $13.50 / 15.90$ & $0.91 / 0.91$ & 73.0/72.0 & $9.00 / 11.00$ & 70 \\
\hline P89 & & $-5.44 /-3.57$ & $\mathrm{PC}_{71} \mathrm{BM} / \mathrm{ITCPT}$ & $1.4 \times 10^{-3}$ & 13.70/17.00 & $0.94 / 0.93$ & 76.0/74.0 & $10.10 / 12.10$ & 70 \\
\hline P90 & & $-5.44 /-3.59$ & $\mathrm{PC}_{71} \mathrm{BM} / \mathrm{ITCPT}$ & & $13.20 / 16.50$ & $0.92 / 0.91$ & 72.0/71.0 & $8.80 / 11.00$ & 70 \\
\hline P91 & & $-5.46 /-3.60$ & $\mathrm{PC}_{71} \mathrm{BM} / \mathrm{ITCPT}$ & $1.1 \times 10^{-3}$ & $13.30 / 15.60$ & $0.92 / 0.91$ & $68.0 / 68.0$ & $8.60 / 10.10$ & 70 \\
\hline P92 & 1.78 & $-5.47 /-3.54$ & $\mathrm{~m}-\mathrm{ITIC}$ & & 16.27 & 0.93 & 60.0 & 9.08 & 71 \\
\hline P93 & 1.77 & $-5.45 /-3.54$ & $\mathrm{~m}-\mathrm{ITIC}$ & & 17.53 & 0.91 & 69.8 & 11.17 & 71 \\
\hline P94 & 1.78 & $-5.44 /-3.54$ & $\mathrm{~m}-\mathrm{ITIC}$ & & 17.06 & 0.90 & 58.5 & 8.99 & 71 \\
\hline P95 & 1.77 & $-5.43 /-3.56$ & $\mathrm{~m}-\mathrm{ITIC}$ & & 16.81 & 0.88 & 54.3 & 8.09 & 71 \\
\hline P96 & 1.98 & $-5.42 /-3.44$ & ITIC & & 15.59 & 0.97 & 54.3 & 8.22 & 72 \\
\hline P97 & 1.87 & $-5.38 /-3.51$ & ITIC & & 15.76 & 0.95 & 59.7 & 8.95 & 72 \\
\hline P98 & 1.83 & $-5.37 /-3.54$ & ITIC & $5.2 \times 10^{-5}$ & 17.18 & 0.94 & 63.5 & 10.26 & 72 \\
\hline P99 & 1.82 & $-5.37 /-3.55$ & ITIC & & 17.19 & 0.90 & 61.7 & 9.56 & 72 \\
\hline P100 & 1.80 & $-5.34 /-3.54$ & ITIC & & 16.30 & 0.88 & 61.4 & 8.82 & 72 \\
\hline P101 & 1.82 & $-5.45 /-3.64$ & IT-4F & $9.75 \times 10^{-5}$ & 21.50 & 0.89 & 78.0 & 15.10 & 73 \\
\hline P102 & 1.83 & $-5.48 /-3.63$ & IT-4F & $9.85 \times 10^{-5}$ & & & & & 73 \\
\hline P103 & 1.87 & $-5.51 /-3.62$ & $\mathrm{IT}-4 \mathrm{~F}$ & $7.91 \times 10^{-5}$ & & & & & 73 \\
\hline P104 & 1.93 & $-5.55 /-3.61$ & IT-4F & $6.63 \times 10^{-5}$ & & & & & 73 \\
\hline P105 & 1.99 & $-5.60 /-3.57$ & $\mathrm{IT}-4 \mathrm{~F}$ & $8.86 \times 10^{-5}$ & & & & & 73 \\
\hline
\end{tabular}

efficient photovoltaic performance. The characteristics and the corresponding device performances of ternary and terpolymer-based OPVs are listed in Table 3.

\section{Polymer donors based on BDD-like acceptor units}

Many BDD-based conjugated polymers with excellent efficiencies have been demonstrated in the aforementioned text. However, rational molecular design is still a challenge to performance, cost, and large-scale fabrication processes. Recently, a few heterocycles, which are structural analogues of the BDD, have been studied as alternative electron-withdrawing candidates in D-A copolymer donors (Fig. 14). Naphtho[2,3-c]thiophene4,9-dione (NTDO), a BDD fused-ring derivative featuring a planar molecular structure and strong electron-deficient properties, has received much attention owing to its promising utilization in OPVs. Cui et al. synthesized the P106 copolymer by introducing DTS units and NTDO units into D-A copolymers ${ }^{12}$. The HOMO levels of P106 decreased to $-5.42 \mathrm{eV}$ and exhibited a preliminary PCE of $5.21 \%$. Aso and coworkers introduced multiple alkyl groups as side chains of the NTDO unit to compare the photovoltaic properties of P107, P108, P109, and P110 ${ }^{74}$. The P108 and P109 copolymers showed improved photovoltaic performances with PCEs over 5\%. Furthermore, by controlling the film morphology using a ternary solvent system, they successfully demonstrated that the PCE of $\mathbf{P 1 0 8} / \mathrm{PC}_{71} \mathrm{BM}$ solar cells further increased to $7.85 \%$. However, for P107 and P110, the reduction of the PCE originated from poor intermixing between the donor and acceptor and led to suppressed exciton dissociation, thus reducing $J_{\mathrm{sc}}$. To investigate the impact of fluorine atoms 


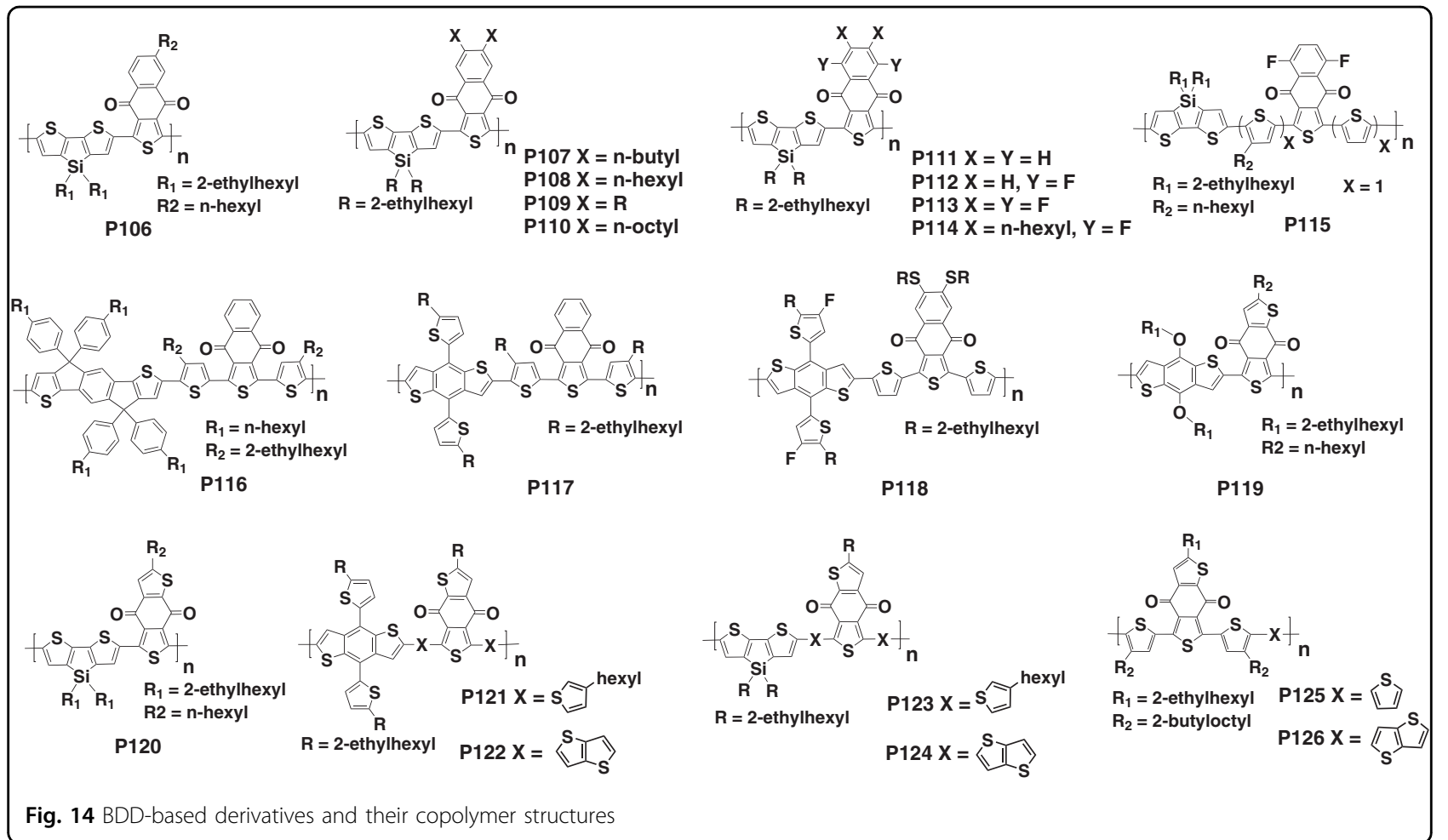

on NTDO-based photovoltaic polymers, Aso et al. designed a series of copolymers (P111, P112, P113, and P114) by introducing different fluorine atoms into the NTDO unit ${ }^{75}$. They found that the D-A characteristics were increased by increasing the number of fluorine atoms and that the HOMO and LUMO levels of the copolymers were fine-tuned depending on the acceptor units. Unexpectedly, on the one hand, the $V_{\text {oc }}$ of $\mathbf{P 1 1 2}$ containing fluorine atoms was lower than that of P108 without fluorination; on the other hand, all these blend films showed an amorphous character and thus led to a moderate PCE of $5.28 \%$. To overcome these drawbacks of fluoro-substituted P112, they further introduced a 3hexylthiophene spacer into the conjugated backbone. The resulting polymer P115 had an enhanced PCE of 9.12\% and $J_{\mathrm{sc}}$ of $17.72 \mathrm{~mA} \mathrm{~cm}^{-2}$ compared with those of $\mathbf{P 1 1 2}^{76}$. Meanwhile, the GIWAS data illustrated the amorphous properties of P115 in both pure and blend films. Therefore, this result showed great promise in developing amorphous OPVs since amorphous systems have high potential for fast deposition and the morphology is not sensitive to the processing conditions.

In addition, Hou et al. investigated two new D-A copolymers called P116 and P117 based on different D units and NTDO as the A unit to evaluate their photovoltaic performance ${ }^{77}$. It was found that the alkylphenyl side groups of $\mathbf{P 1 1 6}$ generated larger torsional dihedral angles and steric hindrance, impeding the interchain $\pi-\pi$ interactions. Moreover, the same structural segment between the donor of P116 and the acceptor of ITIC resulted in a stronger miscibility in the blend, which made it difficult to facilitate the phase separation. Hence, a poor PCE of approximately $4.9 \%$ was obtained in the device based on P116. In contrast, the device based on P117 displayed a clear phase separation in the blend film, which led to a higher device PCE of $7.2 \%$. Cui et al. developed a new NTDO copolymer called P118 by attaching bialkylthio chains onto the NTDO $\mathrm{unit}^{78}$. The copolymer had a deep HOMO level of -5.48 $\mathrm{eV}$, leading to a high $V_{\text {oc }}$ of $0.891 \mathrm{~V}$. The device-based P118 possessed an excellent PCE of $13.10 \%$ and good long-term stability. The initial PCE could remain over $80 \%$ after 2400 hours in air.

As one of the BDD-like A units, benzo[1,2-b:4,5-c'] -dithiophene-4,8-dione (BDTD) possessed more electron deficiency and a stable quinoidal structure, thus reducing the bandgap. In 2012, Ding et al. reported two D-A copolymers P119 and P120 by introducing a BDT unit or DTS unit as an electron-donating D unit and a BDTD unit as an electron-deficient $\mathrm{A}$ unit ${ }^{79}$. As a result, the HOMO level of these two copolymers led to a high $V_{\text {oc }}$ over $0.85 \mathrm{~V}$. In addition, P120 exhibited a better PCE (4.33\%) than that of P119 (2.18\%), resulting from the long wavelength region absorption of P120 compared with that of P119. To further regulate the stereo-structure and photovoltaic performance of copolymers, Li et al. introduced alkylthiophene 
Table 4 Polymer characteristics and the corresponding device performances of BDD-derivative-based OPVs

\begin{tabular}{|c|c|c|c|c|c|c|c|c|c|}
\hline Donor & $E_{\mathrm{g}}^{\mathrm{opt}}(\mathrm{eV})$ & HOMO/LUMO (eV) & Acceptor & $\mu_{\mathrm{h}}\left(\mathrm{cm}^{2} \cdot \mathrm{v}^{-1} \cdot \mathrm{s}^{-1}\right)$ & $J_{s c}\left(\mathrm{~mA} \mathrm{~cm}{ }^{-2}\right)$ & $V_{\text {oc }}(\mathrm{V})$ & FF (\%) & PCE (\%) & ref \\
\hline P106 & 1.65 & $-5.42 /-3.46$ & $\mathrm{PC}_{71} \mathrm{BM}$ & & 9.24 & 0.88 & 64.0 & 5.21 & 12 \\
\hline P107 & 1.63 & $-5.33 /-3.70$ & $P C_{71} B M$ & $7.8 \times 10^{-5}$ & 10.10 & 0.91 & 47.0 & 4.28 & 74 \\
\hline P108 & 1.62 & $-5.25 /-3.63$ & $P C_{71} B M$ & $1.0 \times 10^{-4}$ & 14.39 & 0.86 & 64.0 & 7.85 & 74 \\
\hline P109 & 1.62 & $-5.27 /-3.65$ & $P C_{71} B M$ & $1.1 \times 10^{-4}$ & 9.46 & 0.94 & 58.0 & 5.15 & 74 \\
\hline P110 & 1.64 & $-5.27 /-3.63$ & $P C_{71} B M$ & $9.8 \times 10^{-5}$ & 5.00 & 0.88 & 53.0 & 2.35 & 74 \\
\hline P111 & 1.64 & $-5.37 /-3.73$ & $\mathrm{PC}_{71} \mathrm{BM}$ & $5.1 \times 10^{-5}$ & 8.72 & 0.84 & 35.0 & 2.55 & 75 \\
\hline P112 & 1.59 & $-5.44 /-3.85$ & $P C_{71} B M$ & $1.1 \times 10^{-4}$ & 11.58 & 0.84 & 54.0 & 5.28 & 75 \\
\hline P113 & 1.51 & $-5.59 /-4.07$ & $\mathrm{PC}_{71} \mathrm{BM}$ & $3.5 \times 10^{-5}$ & 4.32 & 0.81 & 28.0 & 0.99 & 75 \\
\hline P114 & 1.65 & $-5.37 /-3.72$ & $P C_{71} B M$ & $3.9 \times 10^{-5}$ & 6.79 & 0.80 & 42.0 & 2.26 & 75 \\
\hline P115 & 1.59 & $-5.29 /-3.70$ & $P C_{71} B M$ & $9.0 \times 10^{-4}$ & 17.72 & 0.77 & 67.0 & 9.12 & 76 \\
\hline P116 & 1.89 & $-5.37 /-3.48$ & $\mathrm{PC}_{71} \mathrm{BM}$ & $2.5 \times 10^{-3}$ & 8.70 & 0.91 & 62.0 & 4.90 & 77 \\
\hline P117 & 1.84 & $-5.37 /-3.53$ & $P C_{71} B M$ & $1.49 \times 10^{-3}$ & 13.00 & 0.89 & 62.0 & 7.20 & 77 \\
\hline P118 & 1.75 & $-5.48 /-3.52$ & $\mathrm{IT}-4 \mathrm{~F}$ & $3.56 \times 10^{-5}$ & 21.03 & 0.89 & 69.9 & 13.10 & 78 \\
\hline P119 & 1.78 & $-5.49 /-3.53$ & $\mathrm{PC}_{61} \mathrm{BM}$ & & 4.75 & 0.87 & 45.0 & 2.18 & 79 \\
\hline P120 & 1.63 & $-5.33 /-3.47$ & $P C_{61} B M$ & & 6.66 & 0.93 & 60.0 & 4.33 & 79 \\
\hline P121 & 1.68 & $-5.30 /-3.78$ & $P C_{71} B M$ & $4.52 \times 10^{-3}$ & 12.81 & 0.81 & 57.3 & 5.91 & 80 \\
\hline P122 & 1.66 & $-5.31 /-3.57$ & $P C_{71} B M$ & $1.84 \times 10^{-5}$ & 6.61 & 0.73 & 63.3 & 3.06 & 80 \\
\hline P123 & 1.78 & $-5.14 /-3.39$ & $P C_{71} B M$ & $1.23 \times 10^{-4}$ & 5.85 & 0.78 & 31.7 & 1.45 & 80 \\
\hline P124 & 1.68 & $-5.18 /-3.59$ & $P C_{71} B M$ & $4.09 \times 10^{-4}$ & 6.47 & 0.74 & 51.2 & 2.45 & 80 \\
\hline P125 & 1.72 & $-5.58 /-3.39$ & $P C_{71} B M$ & $1.28 \times 10^{-4}$ & 10.81 & 0.88 & 66.2 & 6.32 & 81 \\
\hline P126 & 1.61 & $-5.43 /-3.38$ & $P C_{71} B M$ & $1.52 \times 10^{-4}$ & 14.53 & 0.73 & 56.5 & 6.00 & 81 \\
\hline
\end{tabular}

units and thieno[3,2-b]thiophene units as $\pi$-conjugated bridges into the backbones of P119 and P120, building four D- $\pi-A$ copolymers P121, P122, P123, and P124. It was found the HOMO levels of P121 and P123 were approximate, and similar results were also found for $\mathbf{P 1 2 2}$ and $\mathbf{P 1 2 4}{ }^{80}$. This phenomenon indicated that the $\pi$-bridges had more prominent impacts than donor units on the HOMO level in these D- $-\mathrm{A}$ copolymers. As a result, the highest PCE of the four copolymers was still low at approximately $5 \%$, whereas this study clearly proved that $\pi-$ bridges and donor units have a significant influence on their photovoltaic performance. Ding et al. synthesized two D- $\pi-A$ copolymers, $\mathbf{P 1 2 5}$ and P126, using BDTD as an electron-deficient unit, 3-(2-butyloctyl)thienyl as the $\pi$ bridge and thiophene or thienothiophene as the donor unit $^{81}$. Similar photovoltaic performances of P125 and $\mathbf{P 1 2 6}$ were achieved, but the $J_{\text {sc }}$ of P125 was lower than that of P126 because the latter possessed better crystallinity and $\pi-\pi$ stacking, thus promoting hole mobility, rendering the improvement of $J_{\mathrm{sc}}$. Table 4 summarizes the polymer characteristics and the corresponding device performances of BDD-derivative-based OPVs.

\section{Conclusions and perspectives}

In this review, the recent development of polymers based on BDD and its analogue as an electron-accepting unit was systematically summarized, revealing the relationship between their chemical structures and physicochemical as well as photovoltaic properties, including optical absorption, electronic energy level, aggregation property, and photovoltaic performance as donors in OPVs. Meaningfully, this progress provides effective approaches for improving the device performance of OPVs by utilizing various BDD-based polymer donors. The reported results indicate that BDD-based polymers are high-efficiency donors for application in OPVs with fullerene or nonfullerene acceptors and play a very important role in promoting the research progress of OPVs. However, some challenges of the BDD-based polymer donor exist with respect to further increasing the PCE of OPVs by boosting the photocurrent while maintaining a high voltage output. Based on the above discussion, we summarize several approaches for the design and synthesis of outstanding high-performance BDD-based polymer donor materials. 
(1) BDD-based polymer donors with simple molecular structures and fewer synthetic steps to reduce the cost of materials for large-scale commercial applications are designed and synthesized. Currently, the main approaches have been devoted to modulating various donor or acceptor units in BDD-based polymers, including side chain engineering of donor units, fluorination on the $\mathrm{D}-\mathrm{A}$ units or $\pi$-bridges, asymmetry of the polymer backbone, and the use of fused polycyclic groups as the donor units. However, the complicated and high-cost synthetic routes will impede the development of BDD-based polymers. Some simple and low-cost approaches, such as chlorination and steric effects in conjugated blocks, pave a facile route toward large-scale material preparation and commercialization. Meanwhile, BDD analogue acceptor units can also be considered very promising electron-accepting units in constructing efficient polymers if their synthetic cost is low and their energy level and photon harvesting ability can be further improved.

(2) The choice of narrow bandgap nonfullerene acceptors is crucial for obtaining a high PCE, low cost and high stability of the OPVs. A large number of significant efforts and subsequent excellent progress in nonfullerene acceptors, especially the narrow bandgap small molecular acceptors, have witnessed a promising avenue toward high performance.

(3) The terpolymerization strategy shows a feasible and cost-effective method for improving their opticalelectronic properties. Especially for the random terpolymer obtained by combining two electrondonating units with one accepting unit or one electron-donating unit with two electron-accepting units in the same polymer main backbones, the solubility, light absorption and energy levels, crystallinity and blend phase separation can be synergistically tuned to subsequently realize high photovoltaic properties. Therefore, BDD-based materials can be rationally chosen as compatible donor or complement components, which can promote the development of highperformance OPVs.

\section{Acknowledgements}

This work was financially supported by the National Natural Science Foundation of China (NSFC) (No. 21774003) and funded by the Beihang University Youth Talent Support Program (YWF-19-BJ-J-227).

Conflict of interest

The authors declare that they have no conflict of interest.

\section{Publisher's note}

Springer Nature remains neutral with regard to jurisdictional claims in published maps and institutional affiliations.

Received: 4 June 2019 Revised: 16 August 2019 Accepted: 27 August 2019 Published online: 24 January 2020

\section{References}

1. Serap, G., Helmut, N. \& Niyazi, S.S. Conjugated polymer-based organic solar cells. Chem. Rev. 107, 1324-1338 (2007)

2. Barry, C.-T. \& Jean, M.-J.F. Polymer-fullerene composite solar cells. Angew. Chem. Int. Ed. 47, 58-77 (2008)

3. Meng, L.-X. et al. Organic and solution-processed tandem solar cells with 17.3\% efficiency. Science 361, 1094-1098 (2018).

4. Minh, T.-D., Lionel, H., Guillaume, W. \& James, D.-W. Controlling the morphology and performance of bulk heterojunctions in solar cells. Lessons learned from the benchmark poly(3-hexylthiophene):[6,6]-phenyl- $\mathrm{C}_{61}$-butyric acid methyl ester system. Chem. Rev. 113, 3734-3765 (2013).

5. Guo, X. et al. High efficiency polymer solar cells based on poly(3-hexylthiophene)/indeneC70 bisadduct with solvent additive. Energy Environ. Sci. 5, 7943-7949 (2012).

6. Lu, L.-Y. et al. Recent advances in bulk heterojunction polymer solar cells. Chem. Rev. 115, 12666-12731 (2015).

7. Liang, Y.-Y., Xiao, S.-Q., Feng, D.-Q. \& Yu, L.P. Control in energy levels of conjugated polymers for photovoltaic application. J. Phys. Chem. C. 112, 7866-7871 (2008)

8. Li, W. et al. Molecular order control of non-fullerene acceptors for highefficiency polymer solar cells. Joule 3, 1-15 (2019).

9. Agnieszka, P., Philippe, B. \& Mario, L. Thieno[3,4-c]pyrrole-4,6-dione-based polymers for optoelectronic applications. Macromol. Chem. Phys. 214, 7-16 (2013).

10. Yutaka, I. \& Yoshio, A. Development of donor-acceptor copolymers based on dioxocycloalkene-annelated thiophenes as acceptor units for organic photovoltaic materials. Polym. J. 49, 13-22 (2017).

11. Eva, B. \& Frederik, C.-K. Low band gap polymers for organic photovoltaics. Sol. Energy Mater. Sol. Cells 91, 954-985 (2007).

12. Cui, C.H. et al. A D-A copolymer of dithienosilole and a new acceptor unit of naphtho[2,3-c]thiophene-4,9-dione for efficient polymer solar cells. Chem. Commun. 47, 11345-11347 (2011).

13. Yuan, J. et al. Single-junction organic solar cell with over $15 \%$ efficiency using fused-ring acceptor with electron-deficient core. Joule 3, 1140-1151 (2019).

14. Qian, D.P. et al. Synthesis, properties, and photovoltaic performances of donor-acceptor copolymers having dioxocycloalkene-annelated thiophenes as acceptor monomer units. Macromolecules 45, 4564-4571 (2012).

15. Qian, D.P. et al. Design, application, and morphology study of a new photovoltaic polymer with strong aggregation in solution state. Macromolecules 45, 9611-9617 (2012)

16. Yutaka, I., Huang, J.-M., Yasunori, U., Makoto, K. \& Yoshio, A. Synthesis, properties, and photovoltaic performances of donor-acceptor copolymers having dioxocycloalkene-annelated thiophenes as acceptor monomer units. Macromolecules 45, 4564-4571 (2012).

17. Pan, $X$-X. et al. Influence of 2,2-bithiophene and thieno[3,2-b]thiophene units on the photovoltaic performance of benzodithiophene-based wide-bandgap polymers. J. Mater. Chem. C 5, 4471-4479 (2017)

18. Huo, L.-J. et al. Single-junction organic solar cells based on a novel widebandgap polymer with efficiency of 9.7\%. Adv. Mater. 27, 2938-2944 (2015).

19. Wang, Q. et al. Investigations of the conjugated polymers based on dithienogermole (DTG) units for photovoltaic applications. Macromolecules 47 5558-5565 (2014)

20. Huo, L.-J. et al. Organic solar cells based on a 2D benzo[1,2-b:4,5-b']-difuranconjugated polymer with high-power conversion efficiency. Adv. Mater. 27 6969-6975 (2015)

21. Xue, X.-N. et al. Influence of aromatic heterocycle of conjugated side chains on photovoltaic performance of benzodithiophene-based wide-bandgap polymers. Polym. Chem. 7, 4036-4045 (2016).

22. Huang, X.X. et al. Effects of a heteroatomic benzothienothiophenedione acceptor on the properties of a series of wide-bandgap photovoltaic polymers. J. Mater. Chem. C 4, 9052-9059 (2016). 
23. Liu, T. et al. Alkyl side-chain engineering in wide-bandgap copolymers leading to power conversion effciencies over 10\%. Adv. Mater. 29, 1604251 (2017).

24. Huang, W. et al. Molecular engineering on conjugated side chain for polymer solar cells with improved efficiency and accessibility. Chem. Mater. 28, 5887-5895 (2016).

25. Xue, X.-N. et al. Enhanced open-circuit voltage in methoxyl substituted benzodithiophene-based polymer solar cells. Sci. China Chem. 60, 243-250 (2017).

26. Yao, H.F. et al. Dialkylthio substitution: an effective method to modulate the molecular energy levels of 2D-BDT photovoltaic polymers. ACS Appl. Mater. Interfaces 8, 3575-3583 (2016).

27. $\mathrm{Xu}, \mathrm{X}$. et al. Selenium-containing medium bandgap copolymer for bulk heterojunction polymer solar cells with high efficiency of $9.8 \%$. Chem. Mater. 29, 4811-4818 (2017).

28. Cai, Y.-H. et al. High-performance wide-bandgap copolymers based on indacenodithiophene and indacenodithieno[3,2-b]thiophene units. J. Mater. Chem. C 5, 7777-7783 (2017).

29. Gao, X.-D. et al. The regulation of $\pi$-bridge of indacenodithiophene-based donor- $\pi$-acceptor conjugated polymers toward efficient polymer solar cells. Dyes Pigments 162, 43-51 (2019).

30. Qian, D.P. et al. Molecular design toward efficient polymer solar cells with high polymer content. J. Am. Chem. Soc. 135, 8464-8467 (2013).

31. Li, Z.-B. et al. Benzothiadiazole versus thiophene: influence of the auxiliary acceptor on the photovoltaic properties of donor-acceptor-based copolymers. Macromol. Rapid Commun. 39, 1700547 (2017).

32. Fan, B.-B. et al. High-performance conjugated terpolymer-based organic bulk heterojunction solar cells. J. Mater. Chem. A 4, 13930-13937 (2016).

33. Gururaj, P.-K., Jun, Y.-C., Sung, J.-J., Soon, S. \& Doo, K.-M. Controlling the interchain packing and photovoltaic properties via fluorine substitution in terpolymers based on benzo[1,2-c:4,5-c']dithiophene-4,8-dione and benzothiadiazole units. Polymer 148, 330-338 (2018).

34. Yan, C.-Q. et al. Non-fullerene acceptors for organic solar cells. Nat. Rev. Mater 3, 18003 (2018)

35. Cheng, P., Li, G., Zhan, X.-W. \& Yang, Y. Next-generation organic photovoltaics based on non-fullerene acceptors. Nat. Photonics 12, 131-142 (2018).

36. Lin, Y.-Z. \& Zhan, X.-W. Non-fullerene acceptors for organic photovoltaics: an emerging horizon. Mater. Horiz. 1, 470-488 (2014).

37. Xie, D.-J. et al. A novel thiophene-fused ending group enabling an excellent small molecule acceptor for high-performance fullerene-free polymer solar cells with 11.8\% efficiency. Sol. Rrl. 1, 1700044 (2017).

38. Ma, J.-S. et al. A conjugated polymer based on alkylthio-substituted benzo[1,2c:4,5-c]dithiophene-4,8-dione acceptor for polymer solar cells. Dyes Pigments 165, 335-340 (2019).

39. Lin, Y.-Z. et al. An electron acceptor challenging fullerenes for efficient polymer solar cells. Adv. Mater. 27, 1170-1174 (2015).

40. Chen, S.-S. et al. Ultrafast channel II process induced by a 3-D texture with enhanced acceptor order ranges for high-performance non-fullerene polymer solar cells. Energy Environ. Sci. 11, 2569-2580 (2018).

41. Lin, Y.-Z. et al. A facile planar fused-ring electron acceptor for as-cast polymer solar cells with 8.71\% efficiency. J. Am. Chem. Soc. 138, 2973-2976 (2016).

42. Liu, T. et al. High-performance non-fullerene organic solar cells based on a selenium-containing polymer donor and a twisted perylene bisimide acceptor. Adv. Sci. 3, 1600117 (2016).

43. Liu, T. et al. Optimized fibril network morphology by precise side-chain engineering to achieve high-performance bulk-heterojunction organic solar cells. Adv. Mater. 30, 1707353 (2018).

44. Xue, X.-N. et al. Steric engineering of alkylthiolation side chains to finely tune miscibility in nonfullerene polymer solar cells. Adv. Energy Mater. 9, 1802686 (2019).

45. Ye, L., Li, W.-B., Guo, X., Zhang, M.-J. \& Harald, A. Polymer side-chain variation induces microstructural disparity in nonfullerene solar cells. Chem. Mater. https://doi.org/10.1021/acs.chemmater.9b00174 (in press).

46. Li, W.-B. et al. A wide bandgap conjugated polymer donor based on alkoxylfluorophenyl substituted benzodithiophene for high performance nonfullerene polymer solar cells. J. Mater. Chem. A 7, 1307-1314 (2019).

47. Qin, Y.-P. et al. A polymer design strategy toward green solvent processed efficient non-fullerene polymer solar cells. J. Mater. Chem. A 6, 4324-4330 (2018).

48. Li, Y.-H. et al. Design of asymmetric benzodithiophene based wide band-gap conjugated polymers toward efficient polymer solar cells promoted by a low boiling point additive. J. Mater. Chem. C 6, 2806-2813 (2018).
49. Li, Y.-H. et al. Crystalline medium-bandgap light-harvesting donor material based on $\beta$-naphthalene asymmetric-modified benzodithiophene moiety toward efficient polymer solar cells. Chem. Mater. 29, 8249-8257 (2017).

50. Liu, D.-Y. et al. Stirring up acceptor phase and controlling morphology via choosing appropriate rigid aryl rings as lever arms in symmetry-breaking benzodithiophene for high-performance fullerene and fullerene-free polymer solar cells. Adv. Mater. 30, 1705870 (2018).

51. Zhao, W.-C. et al. Molecular optimization enables over $13 \%$ efficiency in organic solar cells. J. Am. Chem. Soc. 139, 7148-7151 (2017).

52. Zhang, S.-Q., Qin, Y.-P., Zhu, J. \& Hou, J.-H. Over $14 \%$ effciency in polymer solar cells enabled by a chlorinated polymer donor. Adv. Mater. 30, 1800868 (2018).

53. Cui, Y. et al. Toward efficient polymer solar cells processed by a solutionprocessed layer-by-layer approach. Adv. Mater. 30, 1802499 (2018).

54. Zhang, S.-Q. et al. A fluorinated polythiophene derivative with stabilized backbone conformation for highly efficient fullerene and non-fullerene polymer solar cells. Macromolecules 49, 2993-3000 (2016).

55. Wu, Y. et al. Realizing enhanced efficiency in nonhalogen solvent processed ternary polymer solar cells by incorporating compatible polymer donor. Sol. Rrl. 2, 1800060 (2018).

56. Ye, L.-L. et al. Insertion of chlorine atoms onto $\pi$-bridges of conjugated polymer enables improved photovoltaic performance. Nano Energy 58, 220-226 (2019).

57. Wu, Y.-N. et al. The crucial role of chlorinated thiophene orientation in conjugated polymers for photovoltaic devices. Angew. Chem. Int. Ed. 57, 12911-12915 (2018).

58. Fei, Z.-P. et al. An alkylated indacenodithieno[3,2-b]thiophene-based nonfullerene acceptor with high crystallinity exhibiting single junction solar cell effciencies greater than 13\% with low voltage losses. Adv. Mater. 30, 1705209 (2018).

59. Flurin, D.-E. et al. Hybridization of local exciton and charge-transfer states reduces nonradiative voltage losses in organic solar cells. J. Am. Chem. Soc. 141, 6362-6374 (2019).

60. Rehman, T. et al. Influence of bridging groups on the photovoltaic properties of wide-bandgap poly(BDTT-alt-BDD)s. ACS Appl. Mater. Interfaces 11, 1394-1401 (2019).

61. Dou, K.-K. et al. Synergistic effect of side-chain and backbone engineering in thieno[2,3-f] benzofuran-based conjugated polymers for high performance nonfullerene organic solar cells. J. Mater. Chem. A 7, 958-964 (2019).

62. Jiang, Z.-Y. et al. Naphtho[1,2-b:5,6-b']dithiophene-based conjugated polymers for fullerene-free inverted polymer solar cells. Macromol. Rapid Commun. 39 1700872 (2018).

63. Wang, X.-C. et al. A maverick asymmetrical backbone with distinct flanked twist angles modulating the molecular aggregation and crystallinity for high performance nonfullerene solar cells. Adv. Energy Mater. 9, 1802530 (2018)

64. Tayebeh, A., Parisa, K., Min, J. \& Christoph, J.-B. Organic ternary solar cells: a review. Adv. Mater. 25, 4245-4266 (2013).

65. An, Q.-S. et al. Effcient ternary organic solar cells with two compatible nonfullerene materials as one alloyed acceptor. Small 8, 1802983 (2018).

66. Liu, T. et al. Highly efficient parallel-like ternary organic solar cells. Chem. Mater 29, 2914-2920 (2017)

67. Liu, T. et al. Ternary organic solar cells based on two highly efficient polymer donors with enhanced power conversion efficiency. Adv. Energy Mater. 6, 1502109(1)-1502109(7) (2015).

68. Lin, Y.-Z. et al. High-performance electron acceptor with thienyl side chains for organic photovoltaics. J. Am. Chem. Soc. 138, 4955-4961 (2016).

69. Kim, J.-Y. et al. Low-temperature processable high-performance D-A-type random copolymers for nonfullerene polymer solar cells and application to flexible devices. Adv. Energy Mater. 8, 1801601 (2018).

70. Huo, L.-J. et al. Subtle side-chain engineering of random terpolymers for high performance organic solar cells. Chem. Mater. 30, 3294-3300 (2018).

71. Jeong, M.-Y. et al. Feasible D1-A-D2-A random copolymers for simultaneous high-performance fullerene and nonfullerene solar cells. Adv. Energy Mater. 8, 1702166 (2018).

72. Hoang, M.H. et al. High-efficiency non-fullerene polymer solar cell fabricated by a simple process using new conjugated terpolymers. J. Mater. Chem. C 7, 111-118 (2019).

73. Cui, Y. et al. Achieving over $15 \%$ effciency in organic photovoltaic cells via copolymer design. Adv. Mater. 31, 1808356 (2019). 
74. Huang, J.-M. et al. Enhanced photovoltaic performance of amorphous copolymers based on dithienosilole and dioxocycloalkene-annelated thiophene. Chem. Mater. 26, 6971-6978 (2014).

75. Yutaka, I. et al. Synthesis, properties, and photovoltaic characteristics of p-type donor copolymers having fluorine-substituted benzodioxocyclohexeneannelated thiophene. J. Mater. Chem. A 5, 19773-19780 (2017).

76. Yutaka, I. et al. Enhanced photovoltaic performance of amorphous donor-acceptor copolymers based on fluorine-substituted benzodioxocyclohexene-annelated thiophene. Adv. Energy Mater. 8, 1702506 (2018).

77. Yang, B. et al. Investigation of conjugated polymers based on naphtho[2,3-c] thiophene-4,9-dione in fullerene-based and fullerene-free polymer solar cells. Macromolecules 50, 1453-1462 (2017).
78. Wu, Y. et al. A new dialkylthio-substituted naphtho[2,3-c]thiophene-4,9-dione based polymer donor for high-performance polymer solar cells. Energy Environ. Sci. 12, 675-683 (2019).

79. Cao, J.-M. et al. Synthesis and photovoltaic properties of low band gap polymers containing benzo[1,2-b:4,5-c']dithiophene-4,8-dione. Macromolecules 45, 1710-1714 (2012)

80. Bin, H.-J., Xiao, L., Liu, Y., Shen, P. \& Li, Y.-F. Effects of donor unit and $\pi-$ bridge on photovoltaic properties of D-A copolymers based on benzo [1,2-b:4,5-c']-dithiophene-4,8-dione acceptor unit. J. Polym. Sci. 52 1929-1940 (2014).

81. Zhao, X.-J., Qian, L., Cao, J.-M., Yan, S.-K. \& Ding, L.-M. D.-A. copolymers with benzo[1,2-b:4,5-c']dithiophene-4,8-dione acceptor unit for polymer solar cells. Polym. Chem. 00, 1-3 (2012). 